\title{
LRLC-shunted piezoelectric vibration absorber
}

Berardengo, M.; Høgsberg, J.; Manzoni, S.; Vanali, M.; Brandt, A.; Godi, T.

Published in:

Journal of Sound and Vibration

Link to article, DOI:

10.1016/j.jsv.2020.115268

Publication date:

2020

Document Version

Peer reviewed version

Link back to DTU Orbit

Citation (APA):

Berardengo, M., Høgsberg, J., Manzoni, S., Vanali, M., Brandt, A., \& Godi, T. (2020). LRLC-shunted piezoelectric vibration absorber. Journal of Sound and Vibration, 474, [115268].

https://doi.org/10.1016/j.jsv.2020.115268

\section{General rights}

Copyright and moral rights for the publications made accessible in the public portal are retained by the authors and/or other copyright owners and it is a condition of accessing publications that users recognise and abide by the legal requirements associated with these rights.

- Users may download and print one copy of any publication from the public portal for the purpose of private study or research.

- You may not further distribute the material or use it for any profit-making activity or commercial gain

- You may freely distribute the URL identifying the publication in the public portal

If you believe that this document breaches copyright please contact us providing details, and we will remove access to the work immediately and investigate your claim 


\title{
LRLC-shunted piezoelectric vibration absorber
}

\author{
M. Berardengo ${ }^{1}$, J. Høgsberg ${ }^{2}$, S. Manzoni ${ }^{3}$, M. Vanali ${ }^{1}$, A. Brandt ${ }^{4}$, T. Godi ${ }^{3}$ \\ 1. Department of Engineering and Architecture - Università degli Studi di Parma \\ Parco Area delle Scienze, 181/A \\ 43124 Parma, Italy \\ 2. Department of Mechanical Engineering - Technical University of Denmark \\ Nils Koppels Allé, building 403 \\ DK-2800 Kongens Lyngby, Denmark \\ 3. Department of Mechanical Engineering - Politecnico di Milano \\ Via La Masa, 34 \\ 20156 Milan, Italy \\ 4. Department of Technology and Innovation - University of Southern Denmark \\ Campusvej 55 \\ DK-5230 Odense M, Denmark
}

\begin{abstract}
This paper addresses a new approach for mono-modal vibration reduction by means of a piezoelectric shunt. It is based on an innovative shunt impedance which allows to improve the attenuation performance and the robustness to mistuning compared to the use of the classical resonant shunt. This result is achieved by building a network, composed of two inductances, one capacitance and one resistance, which generates two resonances, instead of the single resonance imposed by the classical resonant shunt. All the theoretical results discussed in the paper are validated by an experimental campaign on a tailored set-up. These tests show a good agreement between theoretical and experimental results and thereby validate the benefits of the new approach.
\end{abstract}

Keywords: piezoelectric shunt, LRLC shunt, resonant shunt, vibration, damping

*marta.berardengo@unipr.it

Email address: (M. Berardengo ${ }^{1}$, J. Høgsberg ${ }^{2}$, S. Manzoni ${ }^{3}$, M. Vanali ${ }^{1}$, A. Brandt $^{4}$, T. Godi ${ }^{3}$ ) 


\section{Introduction}

2 The use of piezoelectric actuators shunted to electric impedances to attenu3 ate vibrations is a widely studied topic and has recently been thoroughly inves4 tigated (e.g. $[1,2,3,4])$. In this control approach, the piezoelectric transducer 5 acts at the same time as a sensor and as an actuator and the layout of the electric impedance depends on the required type of attenuation. As an example, an impedance composed of either the series or parallel connection of an inductance and a resistance (named resonant shunt or LR shunt) is effective for single-mode control $[5,6,7,8,9,10]$. Conversely, in case of multi-mode control, the use of a resistance coupled to one or two negative capacitances offers good performance $[11,12,13,14]$. Furthermore, other approaches, based on different and more complex networks, are also possible, as shown in $[15,16]$.

Among all the possible approaches, the use of an LR shunt (even with coupling to negative capacitances $[17,18]$ ) has been found to be the most effective approach for controlling a single mode. Different methods have been proposed in the literature to set the values of the inductance $L$ and the resistance $R$ [19]. They can rely on the shape of the frequency response function (FRF) of the controlled system $[6,8]$ or exploit the pole placement theory, requiring defined conditions on the damping associated with the poles of the system [20, 21, 22]. 20 Among the approaches of the latter category, the so-called balanced calibra${ }_{21}$ tion stands out for desirable features such as its high attenuation levels and 22 good robustness to possible mistuning thanks to the way of setting the value ${ }^{23}$ of the resistance $R[21,22]$. The robustness of the control is very important 24 in LR shunts because this type of control suffers from significant performance 25 loss because of mistuning [19, 23, 24]. Indeed, the effect of the LR shunt can

be seen as equivalent to that of tuned vibration absorbers (TVAs), with all the related advantages and drawbacks, such as sensitivity to possible mistuning and parameter uncertainties.

To overcome this limitation, this paper presents a new shunt impedance which uses similar principles as those adopted in [21,22] for the balanced cali- 
bration, while improving the attenuation performance both in tuning and with respect to mistuning. This is achieved by adding a capacitance and an inductance to the existing LR shunt (LRLC shunt, see Sections 3.1 and 3.2) to create an additional resonance for the whole electro-mechanical system, compared to the LR shunt (i.e. the LRLC shunt introduces two resonances in the system, while the LR shunt just one). This LRLC shunt is found to improve both the robustness and performance of the control action.

This approach already showed to be effective in the field of mechanical TVAs, where multiple TVAs are used to add more than one eigenfrequency to the whole system $[25,26]$ and are tuned for attenuating the vibrations of a single mode of the primary system. The technique, applied in different fields, such as for example civil engineering [27, 28, 29, 30] and acoustic control [31], has proven to be able to improve the attenuation provided by a single TVA. TVAs can be used with different layouts, such as series (e.g. [32, 33, 34]), parallel (e.g. $[33,34,35,36,37,38])$ or other configurations (e.g. [39, 40]). Moreover, it is possible to base this technique on smart materials or electro-magnetic interactions (e.g. [41, 42, 43]), which allow to develop new configurations for devices able to add more than one eigenfrequency to the whole system. Unfortunately, most of the time, when smart materials are employed, the obtained equivalent mechanical schemes are different from those typical of multiple TVAs and thus, in these cases, specific optimisation procedures are needed to set the values of all the elements in the control system. Oftentimes, due to the complexity of the problem with additional resonances, these techniques rely on either numerical minimisation of target functions, without analytical formulas, or the numerical solution of a system of polynomial equations and the consequent numerical analysis of the obtained solutions.

The idea of the present work is to exploit the special features of piezoelectric materials for developing an LRLC shunt impedance that adds two eigenfrequencies to the whole system and aims at improving the attenuation performance and robustness of the resonant shunt. To do this, a specific procedure based on a mixed analytical-numerical approach is proposed for the tuning of the elec- 


\section{The system model and the balanced calibration}

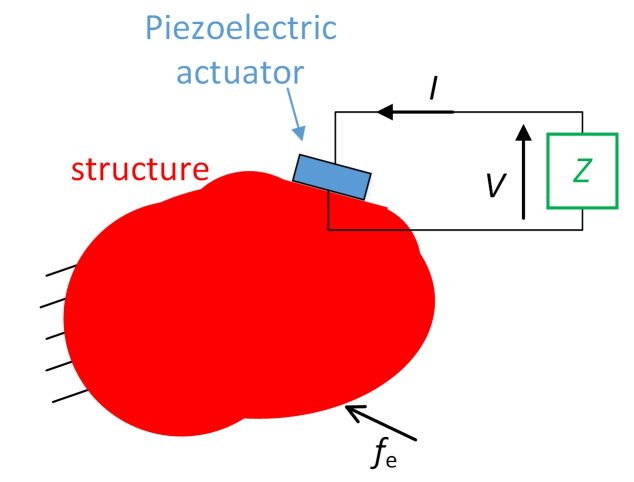

Figure 1: Vibration attenuation by means of a piezoelectric shunt.

tric parameters, providing closed analytical formulations for the basic circuit components.

The structure of the paper is as follows: Section 2 presents the model used for describing the electro-mechanical structure and recalls the balanced calibration approach for setting the values of $L$ and $R$ presented in [21, 22] for the traditional LR shunt. Section 3 presents the new shunt impedance together with its theoretical discussion. Section 4 describes a numerical case aimed at showing the advantages provided by the proposed approach and Section 5 explains how to predict the attenuation that it provides. Finally, Section 6 addresses the experiments carried out to validate the theoretical outcome.$$
Z
$$

$$
\text { (7) }
$$

\footnotetext{
of any given degree-of-freedom $x$ of the structure at time $t$ can be represented
}

in modal coordinates: 
(a)

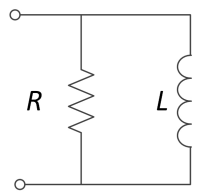

(b)

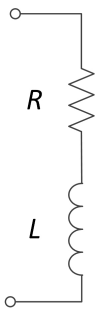

(c)

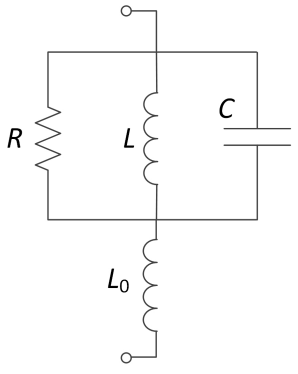

(d)

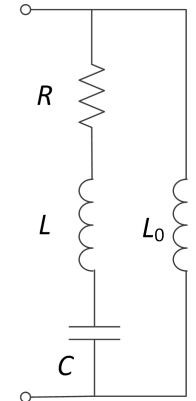

Figure 2: Traditional resonant shunt impedance in parallel (a) and series (b), and the new LRLC shunt impedance in parallel (c) and series (d).

$$
U(x, t)=\sum_{s=1}^{N} \phi_{s}(x) u_{s}(t)
$$

where $N$ is the number of modes of the system, $\phi_{s}$ is the $s$-th eigenvector (scaled to the unit modal mass and with the piezoelectric actuator short-circuited) and $u_{s}$ is the $s$-th modal coordinate.

In case of low modal coupling, the motion of the system for $\omega \simeq \omega_{s}$ (where $\omega$ is the angular frequency and $\omega_{s}$ is the $s$-th eigenfrequency of the system with the piezoelectric actuator short-circuited) can be approximated as:

$$
U(x, t) \simeq \phi_{s}(x) u_{s}(t)
$$

and the equation of motion of the structure becomes:

$$
\left(-\omega^{2} m_{s}+\mathrm{i} \omega c_{s}+k_{s}\right) u_{s}+f=f_{\mathrm{e}, \mathrm{s}}
$$

where $m_{s}, c_{s}$ and $k_{s}$ are the modal mass, damping and stiffness $\left(\omega_{s}=\sqrt{k_{s} / m_{s}}\right)$, respectively, and $f_{\mathrm{e}, \mathrm{s}}$ is the modal forcing. Furthermore, $\mathrm{i}$ is the imaginary unit. From here on, the modal mass will be set equal to $1\left(m_{s}=1\right)$ and thus the eigenvector components are scaled to unit modal mass, as mentioned previously. Furthermore, $f$ is the force exerted by the piezoelectric transducer on the structure and it is expressed as: 


$$
f=\theta_{s} V
$$

where $\theta_{s}$ is the coupling coefficient and $V$ is the voltage across the electrodes of the piezoelectric actuator (see Fig. 1).

The sensor equation couples the electric and mechanical behaviours:

$$
Q=-\theta_{s} u_{s}+C_{s} V
$$

where the charge $Q$ on the transducer surfaces depends on two contributions: the mechanical deformation $\left(-\theta_{s} u_{s}\right)$ and the capacitive effect $\left(C_{s} V\right)$. For vibration damping of a flexible structure with multiple modes, the modal capacitance $C_{s}=C_{0}+C_{s}^{\prime}$ is composed of two contributions: the capacitance associated with constrained transducer boundaries $C_{0}$, and a static correction term $C_{s}^{\prime}$ accounting for the contribution from higher modes [44, 45].

If the shunt impedance $Z$ is considered as in Fig. 1, the equation linking the charge on the surfaces of the piezoelectric transducer and the voltage across its terminals can be written as:

$$
V=-Z(\omega) I=-\mathrm{i} \omega Z(\omega) Q
$$

where $I$ is the current flowing in the circuit (see Fig. 1). According to the type of shunt impedance used, the expression of $Z$ changes. If the classical LR shunt is considered, $Z$ either represents the series or parallel connection of a resistance $R$ and an inductance $L$. These specific cases are shown in Figs. 2a and $\mathrm{b}$ and treated in the next subsection.

\subsection{The classical resonant shunt and its balanced calibration}

Relying on the electrical analogy of mechanical systems and knowing that an electrical series connection corresponds to a parallel mechanical connection and vice versa, it is possible to translate the electrical model of Fig. 3a ( $L$ and $R$ connected in parallel) into an equivalent mechanical model, as shown in Fig. 3b ( $m$ and $c$ in series). In this equivalent representation, the mechanical parameters 

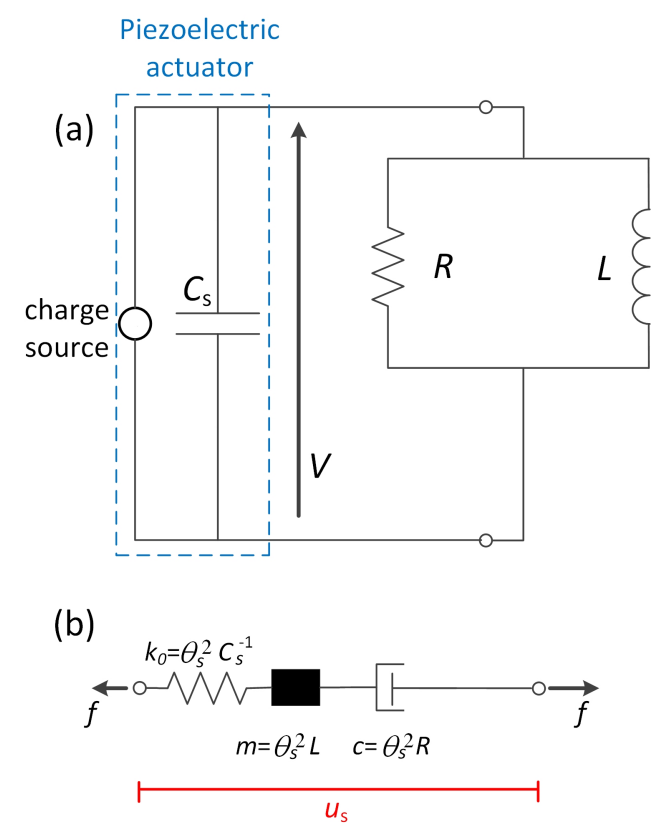

Figure 3: Circuit diagram of the parallel LR shunt (a) and its mechanical equivalent (b).

are obtained by multiplying the electrical parameters by $\theta_{s}^{2}$. A similar approach can be adopted for the series connection between $L$ and $R$ (see Figs. 4a and b).

Using Eqs. (4), (5) and (6) in Eq. (3), the FRF of the electro-mechanical system is obtained. According to the type of connection between inductance $L$ and resistance $R$ (i.e. parallel or series in Figs. 2a and b, respectively), the mathematical description of $Z$ changes and two different FRFs are achieved. In the case of a parallel link, the FRF is:

$\frac{u_{s} k_{s}}{f_{\mathrm{e}, \mathrm{s}}}=\frac{k_{s}\left(-\omega^{2}+2 \mathrm{i} \zeta_{\mathrm{e}} \omega_{\mathrm{e}} \omega+\omega_{\mathrm{e}}^{2}\right)}{\left[-\omega^{2}+2 \mathrm{i} \zeta_{\mathrm{s}} \omega_{\mathrm{s}} \omega+\left(1+\kappa_{0}^{2}\right) \omega_{\mathrm{s}}^{2}\right]\left(-\omega^{2}+2 \mathrm{i} \zeta_{\mathrm{e}} \omega_{\mathrm{e}} \omega+\omega_{\mathrm{e}}^{2}\right)-\kappa_{0} \omega_{\mathrm{s}}^{2}\left(\omega_{\mathrm{e}}^{2}+2 \mathrm{i} \zeta_{\mathrm{e}} \omega_{\mathrm{e}} \omega\right)}$

122 while in case of a series link, the FRF is instead given as:

$$
\frac{u_{s} k_{s}}{f_{\mathrm{e}, \mathrm{s}}}=\frac{k_{s}\left(-\omega^{2}+2 \mathrm{i} \zeta_{\mathrm{e}} \omega_{\mathrm{e}} \omega+\omega_{\mathrm{e}}^{2}\right)}{\left[-\omega^{2}+2 \mathrm{i} \zeta_{\mathrm{s}} \omega_{\mathrm{s}} \omega+\left(1+\kappa_{0}^{2}\right) \omega_{\mathrm{s}}^{2}\right]\left(-\omega^{2}+2 \mathrm{i} \zeta_{\mathrm{e}} \omega_{\mathrm{e}} \omega+\omega_{\mathrm{e}}^{2}\right)-\kappa_{0} \omega_{\mathrm{s}}^{2} \omega^{2}}
$$

${ }_{123}$ The symbol $\zeta_{s}$ indicates the non-dimensional damping ratio associated with the 


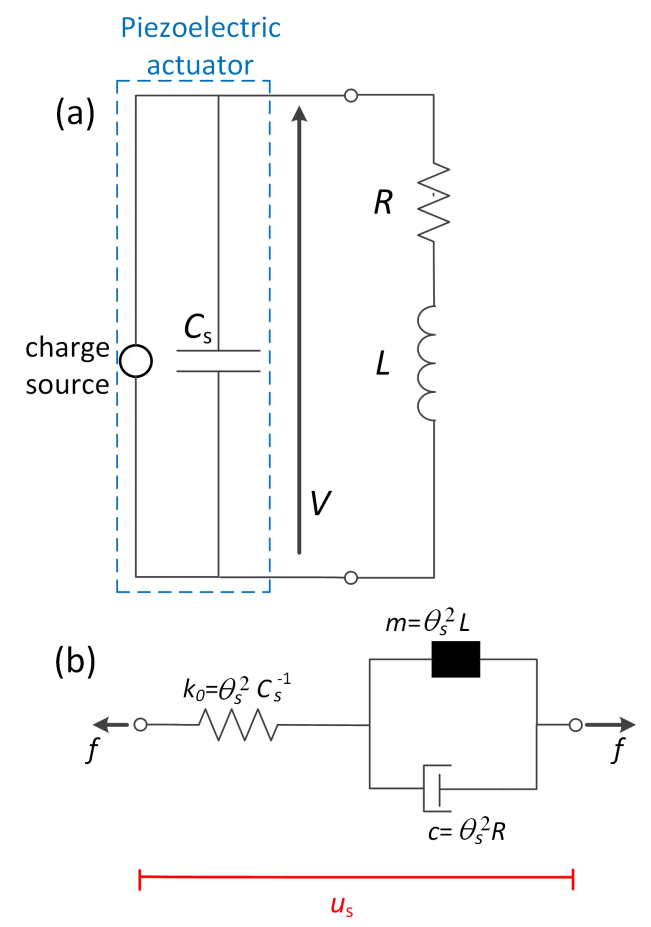

Figure 4: Circuit diagram of the series LR shunt (a) and its mechanical equivalent (b).

structural eigenfrequency $\omega_{s}$, while $\omega_{\mathrm{e}}$ and $\zeta_{\mathrm{e}}$ are the electric eigenfrequency and non-dimensional damping ratio, respectively. The electric eigenfrequency $\omega_{\mathrm{e}}$ is related to $L$ by the following relation:

$$
\omega_{\mathrm{e}}=\frac{1}{\sqrt{L C_{s}}}
$$

The expression of $\zeta_{\mathrm{e}}$ depends on the connection of $L$ and $R$. For the parallel connection, its expression is:

$$
\zeta_{\mathrm{e}}=\frac{1}{2 \omega_{\mathrm{e}} R C_{s}}
$$

129 whereas for the series connection, it is given as:

$$
\zeta_{\mathrm{e}}=\frac{R}{2 \omega_{\mathrm{e}} L}
$$


Moreover, a normalised coupling coefficient $\kappa_{0}$ is expressed as (see Figs. 3b and $4 b)$ :

$$
\kappa_{0}=\frac{k_{0}}{k_{s}}=\frac{\theta_{s}^{2}}{C_{s} k_{s}}
$$

It is noted that $\sqrt{\kappa_{0}}$ is the modal electro-mechanical coupling coefficient [21], which can be estimated as [44, 46, 47]:

$$
\kappa_{0}=\frac{\theta_{s}^{2}}{C_{s} \omega_{s}^{2}} \simeq \frac{\hat{\omega}_{s}^{2}-\omega_{s}^{2}}{\omega_{s}^{2}}
$$

where $\hat{\omega}_{s}$ is the system eigenfrequency for the piezoelectric actuator with opencircuited electrodes.

The FRFs in Eqs. (7) and (8) are characterised by four poles. More precisely, for low to moderate damping values, they appear as two pairs of complex conjugate eigenvalues. The balanced calibration, considered here as the starting point of the proposed method, is based on the requirement of equal modal damping of the eigenvalues. This tuning approach for the shunt impedance has already demonstrated to provide simultaneously high attenuation values (close to those provided by minimisation criteria on the FRF amplitude) and a high robustness to possible mistuning due to an increased value of $R$ (see e.g. [21]).

As demonstrated in $[21,48,49]$, plotting the absolute value of the real and imaginary parts of the eigenvalues (normalised by a real-valued reference frequency $\omega_{0}$ ) is a good way to investigate whether the condition of equal modal damping is fulfilled. Indeed, the condition of equal modal damping is achieved when the normalised eigenvalues lie on the same line containing the origin of the complex plane (plotting them in terms of absolute value of the real and imaginary parts) and thereby appear as inverse points with respect to a unit circle. The value of $\omega_{0}$ is automatically obtained when the equal modal damping condition is imposed (see [21]) and represents the anti-resonance frequency in the FRF when $\zeta_{e}=0$. For the parallel connection of $L$ and $R$, the reference frequency is: 


$$
\omega_{0}=\omega_{s}
$$

while, for the series connection, it is:

$$
\omega_{0}=\omega_{s} \sqrt{1+\kappa_{0}}
$$

Following this tuning procedure, it is thus possible to derive the expressions of $\omega_{\mathrm{e}}$ and $\zeta_{\mathrm{e}}$ which secure equal modal damping, denoted as $\omega_{\mathrm{e}}^{\mathrm{opt}}$ and $\zeta_{\mathrm{e}}^{\mathrm{opt}}$, respectively. For the parallel connection of $L$ and $R$, they are:

$$
\begin{gathered}
\omega_{\mathrm{e}}^{\mathrm{opt}}=\omega_{s} \\
\zeta_{\mathrm{e}}^{\mathrm{opt}}=\sqrt{\frac{\kappa_{0}}{2}}
\end{gathered}
$$

while, for the series connection, their expressions are:

$$
\begin{gathered}
\omega_{\mathrm{e}}^{\mathrm{opt}}=\omega_{s}\left(1+\kappa_{0}\right) \\
\zeta_{\mathrm{e}}^{\mathrm{opt}}=\sqrt{\frac{\kappa_{0}}{2\left(1+\kappa_{0}\right)}}
\end{gathered}
$$

From this tuning procedure, obtaining a robust LR shunt, the authors propose a new layout for the shunt impedance $Z$, composed of two inductances $(L$ and $L_{0}$ ), a capacitance $C$ and a resistance $R$ (see Figs. 2c and d). It is conceived to further improve the performance and the robustness of the LR shunt by introducing an additional resonance in the system. As mentioned, it is referred to as an LRLC shunt and introduced in the next section.

\section{The LRLC shunt}

The LRLC shunt proposed in this paper is calibrated based on the requirement of equal modal damping, as introduced for the classical resonant shunt in Section 2.1. The shunt circuit, its coupling with the electro-mechanical structure and its tuning procedure are presented in this section. Particularly, two 
different electrical circuits will be considered. The parallel LRLC and the series LRLC, discussed in Sections 3.1 and 3.2, respectively. The absorber system is similar to a mechanical vibration absorber, suspended by either a shunted piezoelectric [50] or electromagnetic [43] transducer. However, in the present case, the absorber is realized entirely by a shunt and thus without a physical vibratory mass.

\subsection{The parallel $L R L C$}

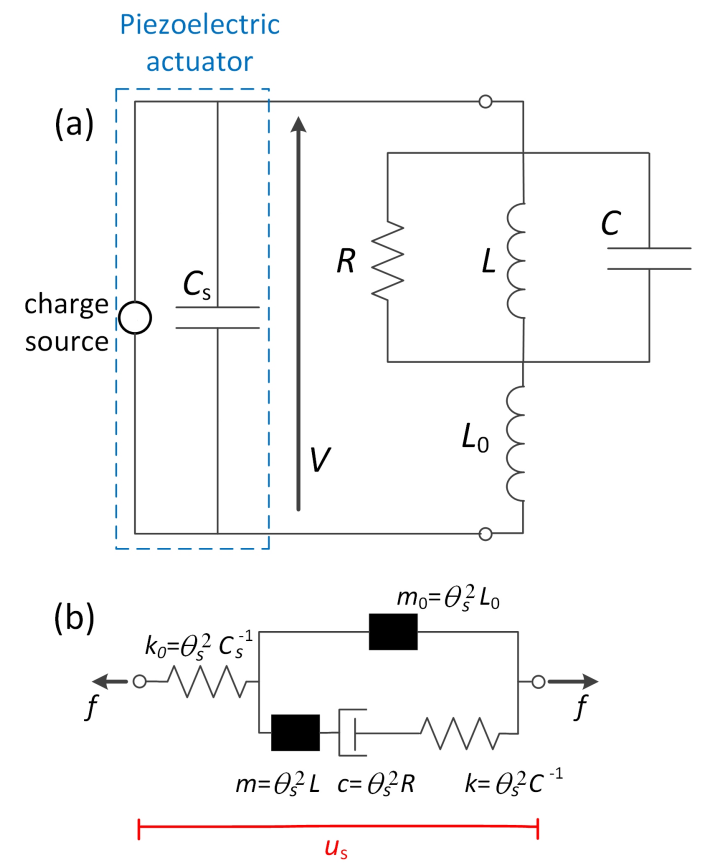

Figure 5: Circuit diagram of the parallel LRLC shunt (a) and its mechanical equivalent (b).

The shunt impedance discussed in this section has the layout shown in Fig. 2c. When it is connected to the piezoelectric actuator, the electrical model of Fig. 5a is obtained. Figure 5a shows that a null value of $R$ short-circuits its branch. Therefore, no current flows through $L$ and $C$ (i.e. the current flows through the short-circuited branch) and, consequently, only a single resonance is created by the transducer capacitance $C_{s}$ and the leading shunt inductance $L_{0}$. 
Conversely, when $R$ is not null, current flows through the $L$ and $C$ branches and, thus, an additional resonance is introduced. In comparison with the classical LR shunt, the proposed layout adds a supplementary resonance that will improve performance and robustness, when calibrated properly.

\subsubsection{FRF}

As already mentioned for the LR shunt, using the impedance analogy between electrical and mechanical systems, the electrical model of Fig. 5a may be translated into the equivalent mechanical model shown in Fig. 5b. The applied electro-mechanical equivalence is similar to that employed in the previously referenced works $[21,22]$ related to the balanced calibration of the LR shunt, in order to obtain a parallelism between the analytical treatments. Thus, the mechanical parameters are again simply obtained by multiplying the electrical parameters by $\theta_{s}^{2}$.

Using the approach employed in Section 2.1, and taking into account the parallel impedance layout in Eq. (6), the FRF of the parallel LRLC system can be derived as:

$$
\frac{u_{s} k_{s}}{f_{\mathrm{e}, \mathrm{s}}}=\frac{\left(-r^{2} \mu_{0}+\kappa_{0}\right) G-r^{2} \mu \kappa}{\left(-r^{2}+2 \mathrm{i} r \zeta_{s}+1\right)\left\{\left(-r^{2} \mu_{0}+\kappa_{0}\right) G-r^{2} \mu \kappa\right\}-r^{2} \kappa_{0}\left(\mu_{0} G+\kappa \mu\right)}
$$

where the frequency function $G$ for the parallel circuit in Fig. 5a is:

$$
G=-r^{2} \mu+\mathrm{i} r \frac{\mu \kappa}{\beta}+\kappa
$$

while $r$ is the normalised frequency:

$$
r=\frac{\omega}{\omega_{s}}
$$

The remaining system ratios in Eq. (20) are:

$$
\kappa_{0}=\frac{k_{0}}{k_{s}}, \mu_{0}=\frac{m_{0}}{m_{s}}, \kappa=\frac{k}{k_{s}}, \mu=\frac{m}{m_{s}}, \beta=\frac{c}{\sqrt{m_{s} k_{s}}}
$$

where $m_{0}, k_{0}, m, c$ and $k$ are the parameters of the equivalent mechanical representation of the LRLC circuit, as defined in Fig. 5b. 
$\left[\omega^{4}-2\left(1+2 \chi^{2}\right) \omega_{0}^{2} \omega^{2}+\omega_{0}^{4}-4 \mathrm{i} \chi \tau \omega_{0} \omega\left(\omega^{2}-\omega_{0}^{2}\right)\right]\left(-\omega^{2}+2 \mathrm{i} \zeta_{3} \omega_{0} \omega+\omega_{0}^{2}\right)=0(26)$

226 Equation (26) can also be expressed as a function of the dimensionless root ${ }_{227} \xi=\omega / \omega_{0}=r / \Omega_{0}$ with $\Omega_{0}=\omega_{0} / \omega_{\mathrm{s}}$, leading to:

where $\chi$ and $\tau$ are parameters depending on the frequency and the damping associated with the eigenvalues.

The second requirement on the pole positions is to have the last pair of poles at a frequency value equal to $\omega_{0}$, thus leading to the following polynomial form:

$$
-\omega^{2}+2 \mathrm{i} \zeta_{3} \omega_{0} \omega+\omega_{0}^{2}=0
$$

${ }_{22}$ where $\zeta_{3}$ is the non-dimensional damping ratio associated with the considered eigenvalues. Using the conditions of Eqs. (24) and (25), the following resulting equation can be constructed as: 
Table 1: Conditions on the coefficients of Eq.

\begin{tabular}{cc}
\hline quantity & value \\
\hline ratio between the coefficients of & -1 \\
the 6-th and 0-th order terms of Eq. (27) & 1 \\
\hline \multicolumn{2}{c}{ ratio between the coefficients of } \\
the 5-th and 1-st order terms of Eq. (27) \\
\hline $\begin{array}{l}\text { ratio between the coefficients of } \\
\text { the 4-th and 2-nd order terms of Eq. (27) }\end{array}$ \\
\hline
\end{tabular}

$$
\begin{array}{r}
-\xi^{6}+\xi^{5}\left(2 \mathrm{i} \zeta_{3}+4 \mathrm{i} \chi \tau\right)+\xi^{4}\left[1+8 \zeta_{3} \chi \tau+2\left(1+2 \chi^{2}\right)\right]+ \\
\xi^{3}\left[-8 \mathrm{i} \chi \tau-2 \mathrm{i} \zeta_{3}\left(2+4 \chi^{2}\right)\right]+\xi^{2}\left[-1-8 \zeta_{3} \chi \tau-2\left(1+2 \chi^{2}\right)\right]+ \\
\xi\left(2 \mathrm{i} \zeta_{3}+4 \mathrm{i} \chi \tau\right)+1=0
\end{array}
$$

\subsubsection{Equal damping calibration}

From Eq. (27), it is evident that the condition of equal modal damping for four of the poles and the condition related to the value of the eigenfrequency on the other two translate into the three conditions gathered in Tab. 1.

To derive the tuning conditions it is sufficient to apply the abovementioned requirements about the pole locations to the actual poles of the characteristic polynomial, which can be obtained from the denominator of Eq. (20). Indeed, the denominator of Eq. (20) can be expressed in terms of $\xi$ and posed equal to zero in order to derive the eigenvalues in terms of the lumped physical parameters:

$$
\begin{array}{r}
-\xi^{6}+\xi^{4} \frac{1}{\Omega_{0}^{2}}\left(1+\kappa_{0}+\frac{\kappa+\kappa_{0}}{\mu_{0}}+\frac{\kappa}{\mu}\right)-\xi^{2} \frac{1}{\Omega_{0}^{4}}\left[\frac{\kappa}{\mu}\left(1+\kappa_{0}\right)+\frac{\kappa_{0}}{\mu_{0}}\left(1+\frac{\kappa}{\kappa_{0}}+\frac{\kappa}{\mu}+\kappa\right)\right]+ \\
\frac{\kappa \kappa_{0}}{\Omega_{0}^{6} \mu \mu_{0}}+\mathrm{i} \xi \frac{\kappa}{\Omega_{0} \beta}\left[\xi^{4}-\xi^{2} \frac{1}{\Omega_{0}^{2}}\left(1+\frac{\kappa_{0}}{\mu_{0}}+\kappa_{0}\right)+\frac{\kappa_{0}}{\Omega_{0}^{4} \mu_{0}}\right]=0
\end{array}
$$

Requiring the equality between the coefficients of Eqs. (28) and (27) (thus obtaining six equations: from the 5 -th order to the 0 -th order) allows to find the 
values of the physical electrical parameters, which thereby satisfy the condition of equal modal damping. Three of these equations can be replaced by imposing the three conditions stated in Tab. 1, simplifying the solution process. Applying these three conditions to the coefficients of Eq. (28) (terms of order 0, 1, 2, 4, 5 and 6 ), the following three equations are obtained:

$$
\begin{gathered}
\frac{\kappa \kappa_{0}}{\Omega_{0}^{6} \mu \mu_{0}}=1 \\
\frac{\kappa_{0}}{\Omega_{0}^{4} \mu_{0}}=1 \\
\frac{1}{\Omega_{0}^{2}}\left(1+\kappa_{0}+\frac{\kappa+\kappa_{0}}{\mu_{0}}+\frac{\kappa}{\mu}\right)=\frac{1}{\Omega_{0}^{4}}\left[\frac{\kappa}{\mu}\left(1+\kappa_{0}\right)+\frac{\kappa_{0}}{\mu_{0}}\left(1+\frac{\kappa}{\kappa_{0}}+\frac{\kappa}{\mu}+\kappa\right)\right]
\end{gathered}
$$

whose solution leads to the following parameter relations:

$$
\mu_{0}=\frac{\kappa_{0}}{\left(1+\kappa_{0}\right)^{2}}, \mu=\frac{\kappa}{1+\kappa_{0}}, \Omega_{0}^{2}=1+\kappa_{0}
$$

The value of $\kappa_{0}$ depends on the physical properties of the electro-mechanical system and is therefore considered known, while the value of $\mu_{0}$ (thus $L_{0}$ ) can be readily found from Eq. (32). When deriving the value of $\mu$ (thus $L$ ) as a function of $\kappa$ (thus $C$ ) using Eq. (32), five parameters are still unknown: $\kappa, \beta$, $\zeta_{3}, \chi$ and $\tau$. Three equations out of the six original equations of the problem have been already used to derive Eq. (32) (see Tab. 1). Hence, the problem is overdetermined and two parameters must be chosen by the user or derived by adding additional constraints to the problem. In this paper, it is chosen to formulate an additional control target to derive the values of $\kappa$ and $\beta$. Their tuning procedure is described in the next subsection.

\subsubsection{Amplitude minimisation}

The additional requirement in this case is the minimisation of the $\mathrm{H}_{\infty}$ norm of the FRF. Indeed, the value of $\beta$ that guarantees, for a given value of $\kappa$, the minimisation of the peak of the dynamic amplification (i.e. $\mathrm{H}_{\infty}$ control) has 

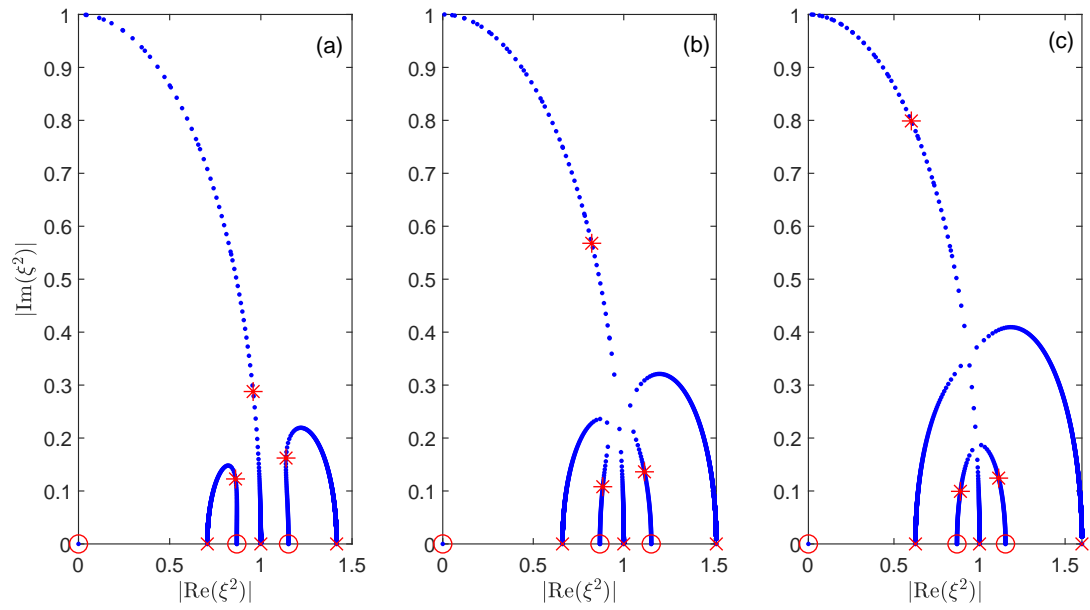

Figure 6: Trends of the absolute values of the real and imaginary parts of the squared roots (LRLC in parallel layout): $\kappa=0.10 \kappa_{0}(\mathrm{a}), \kappa=0.15 \kappa_{0}$ (b) and $\kappa=0.20 \kappa_{0}$ (c). The value of $\kappa_{0}$ is 0.02 .

been chosen in this paper. To find this optimal value, a numerical minimisation of the maximum of the FRF amplitude of the electro-mechanical system must be carried out. Indeed, an analytical solution is not straightforward to be found. It is also noticed that the user can choose the $\beta$ value according to another control target (e.g. $\mathrm{H}_{2}$ control) or to the desired level of vibration mitigation. Once the conditions for equal modal damping are set, providing closed-form analytical formulas for the values of $\mu_{0}$ and $\mu$, the subsequent numerical minimisation finds the optimal $\kappa$ and $\beta$ values according to the desired performance target $\left(\mathrm{H}_{\infty}\right.$ optimisation in this case).

The choice of the optimal $\kappa$ value arises from considerations on the shape of the root locus of the controlled system as a function of this parameter. Figure 6 shows the complex root trajectories in the $\xi^{2}$-plane for three different values of $\kappa / \kappa_{0}$, chosen as an example. In the plots of Fig. 6 , the red crosses indicate the roots for $\beta \rightarrow \infty$, the circles are for $\beta=0$, while the asterisks represent the roots obtained with the optimal $\beta$ value from an $\mathrm{H}_{\infty}$-norm optimisation. As expected from the requirements imposed on the pole positions in Eqs. (27) and (28), one of the squared poles lies on a circle with unit radius, while the other two are 
inverse points with respect to this circle. It is possible to demonstrate that, if the normalised eigenvalues lie on the same line containing the origin of the complex plane, and thus represent inverse points with respect to a circle of unit radius, the same holds for the squared eigenvalues. Looking at the three loci of Fig. 6 (that are all characterised by the equal modal damping condition), it can be noticed that, by decreasing the value of $\kappa / \kappa_{0}$, the trajectories described by two of the squared poles (those not on the circle with unitary radius) separate into two different side lobes (see Fig. 6a) passing through a bifurcation condition (see Fig. 6b), where all three squared poles coincide for a certain value of $\beta$. Although this bifurcation point looks as the best choice from the modal damping point of view, it actually leads to a high modal coupling and thereby to a nonoptimal solution in terms of dynamic amplification, as explained in [21, 48]. Furthermore, a shunt without roots near a bifurcation point in the complex plane is expected to be robust with respect to calibration because the roots are well separated. Therefore, the optimal solution in terms of $\mathrm{H}_{\infty}$ optimisation is achieved with a $\kappa$ value such that the complex roots are sufficiently separated, which is furthermore expected to provide good robustness.

\subsubsection{Bifurcation point}

The condition mentioned above (to guarantee the desired amount of modal damping, while keeping the complex roots sufficiently separated) occurs for a $\kappa$ value that is lower than for the bifurcation point, as indicated by the asterisks in Fig. 6 (optimal by the $\mathrm{H}_{\infty}$ norm of Eq. (20)) and their respective FRF amplitudes shown in Fig. 7. The dynamic amplification for the optimal $\beta$ value and $\kappa=0.1 \kappa_{0}$ (see Fig. 7a) is indeed lower than for the other two cases in Figs. $7 \mathrm{~b}$ and $\mathrm{c}$. From this analysis, it can be concluded that the optimal value of $\kappa$ must be searched numerically among all the values lower than that leading to the bifurcation point of the three squared roots. This threshold $\kappa_{\text {thr,p }}$ can be derived analytically by noticing that all the three squared poles have the same frequency and damping values in the bifurcation point and thus are all placed on the circle with unitary radius in the complex $\xi^{2}$-plane. Therefore, they must 

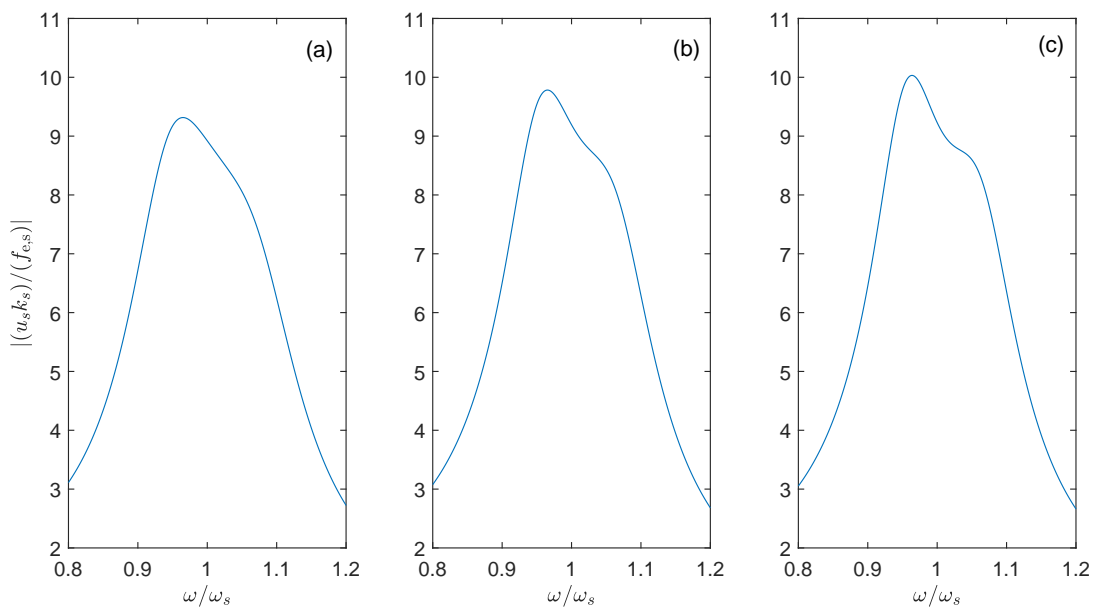

Figure 7: Amplitude of the FRF (LRLC in parallel layout, see Eq. (20)): $\kappa=0.10 \kappa_{0}$ (a), $\kappa=0.15 \kappa_{0}$ (b) and $\kappa=0.20 \kappa_{0}$ (c). The value of $\kappa_{0}$ is 0.02 and the $\beta$ value is set according to the $\mathrm{H}_{\infty}$-norm optimisation (see the asterisks in Fig. 6).

satisfy the following condition:

$$
\begin{array}{r}
\left(-\xi^{2}+2 \mathrm{i} \xi \zeta+1\right)^{3}=0 \Rightarrow-\xi^{6}+6 \zeta \mathrm{i} \xi^{5} \\
+\left(3+12 \zeta^{2}\right) \xi^{4}-\left(12 \zeta+8 \zeta^{3}\right) \mathrm{i} \xi^{3}-\left(3+12 \zeta^{2}\right) \xi^{2}+6 \zeta \mathrm{i} \xi+1=0
\end{array}
$$

If the first, second and third order terms of Eq. (33) are equated to those of Eq. (28), using the expressions in Eq. (32), the value of $\kappa_{\mathrm{thr}, \mathrm{p}}$ is found as:

$$
\kappa_{\mathrm{thr}, \mathrm{p}}=\frac{8 \kappa_{0}^{2}}{1+\kappa_{0}}
$$

The optimal $\kappa$ value is then in the following chosen less than $\kappa_{\text {thr,p }}$ to avoid the bifurcation point (see Section 3.3).

\subsection{The series $L R L C$}

The shunt discussed in this section has the layout shown in Fig. 2d and the approach used to derive the FRF of the controlled system is the same as described for the parallel shunt in Section 3.1. As in the case of the parallel 


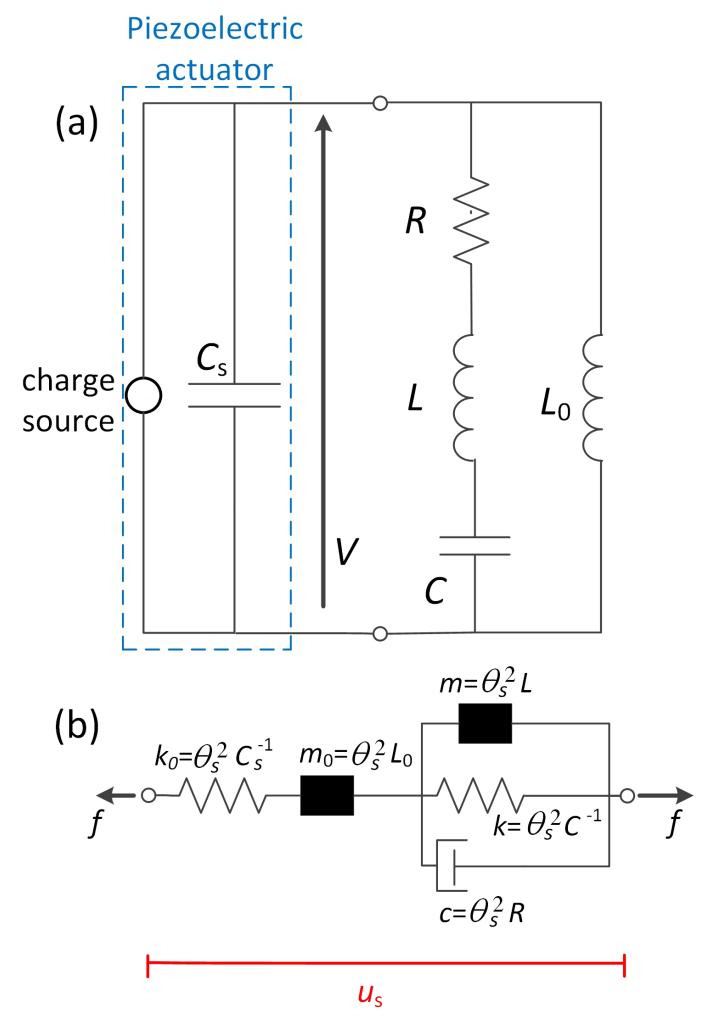

Figure 8: Circuit diagram of the series LRLC shunt (a) and its mechanical equivalent (b).

LRLC, the electrical model of Fig. 8a can be converted into the equivalent mechanical model shown in Fig. 8b.

Figure 8a shows that, for $R \rightarrow \infty$, a single resonance is created by the transducer capacitance $C_{s}$ and the leading shunt inductance $L_{0}$, while, for finite values of $R$ (i.e. decreasing $R$ from $\infty$ to zero), an additional resonance is introduced by the shunt components $C$ and $L$.

\subsubsection{FRF}

Using the same approach as employed in Section 3.1, the FRF of the system can be derived: 


$$
\frac{u_{s} k_{s}}{f_{\mathrm{e}, \mathrm{s}}}=\frac{\left(-r^{2} \mu_{0}+\kappa_{0}\right) E-r^{2} \mu_{0} \kappa_{0}}{\left(-r^{2}+2 \mathrm{i} r \zeta_{s}+1\right)\left\{\left(-r^{2} \mu_{0}+\kappa_{0}\right) E-r^{2} \mu_{0} \kappa_{0}\right\}-r^{2} \kappa_{0} \mu_{0} E}
$$

where the frequency function $E$ is defined as:

$$
E=-r^{2} \mu+\mathrm{i} r \beta+\kappa
$$

The parameters $\kappa_{0}, \mu_{0}, \kappa, \mu$ and $\beta$ are defined as in Eq. (23). Furthermore, by looking at the functions $E$ and $G$ (see Eq. (21)), it is possible to notice that the damping term is defined differently in the series and parallel layouts.

As already mentioned, the optimal shunt parameters are derived from conditions on the system poles. The system eigenvalues can be found by expressing the denominator of the FRF in Eq. (35) as a function of the dimensionless frequency $\xi$ (neglecting the mechanical damping) and letting it equal zero:

$$
\begin{array}{r}
-\xi^{6}+\xi^{4} \frac{1}{\Omega_{0}^{2}}\left(1+\kappa_{0}\left(1+\frac{1}{\mu_{0}}+\frac{1}{\mu}\right)+\frac{\kappa}{\mu}\right)-\xi^{2} \frac{1}{\Omega_{0}^{4}}\left[\frac{\kappa_{0} \kappa}{\mu_{0} \mu}+\kappa_{0}\left(\frac{1}{\mu_{0}}+\frac{1+\kappa}{\mu}\right)+\frac{\kappa}{\mu}\right]+ \\
\frac{\kappa \kappa_{0}}{\Omega_{0}^{6} \mu \mu_{0}}+\mathrm{i} \xi \frac{\beta}{\Omega_{0} \mu}\left[\xi^{4}-\xi^{2} \frac{1}{\Omega_{0}^{2}}\left(1+\kappa_{0}+\frac{\kappa_{0}}{\mu_{0}}\right)+\frac{\kappa_{0}}{\Omega_{0}^{4} \mu_{0}}\right]=0
\end{array}
$$

\subsubsection{Equal damping calibration}

The three conditions of Tab. 1 can then be applied to the coefficients (of the terms of order $0,1,2,4,5$ and 6 ) of Eq. (37) to require equal modal damping, leading to the following three equations:

$$
\begin{gathered}
\frac{\kappa \kappa_{0}}{\Omega_{0}^{6} \mu \mu_{0}}=1 \\
\frac{\kappa_{0}}{\Omega_{0}^{4} \mu_{0}}=1 \\
\frac{1}{\Omega_{0}^{2}}\left(1+\kappa_{0}\left(1+\frac{1}{\mu_{0}}+\frac{1}{\mu}\right)+\frac{\kappa}{\mu}\right)=\frac{1}{\Omega_{0}^{4}}\left[\frac{\kappa_{0} \kappa}{\mu_{0} \mu}+\kappa_{0}\left(\frac{1}{\mu_{0}}+\frac{1+\kappa}{\mu}\right)+\frac{\kappa}{\mu}\right]
\end{gathered}
$$

The solution to these equations gives the following three parameter relations: 


$$
\mu_{0}=\kappa_{0}, \mu=\kappa, \Omega_{0}^{2}=1
$$

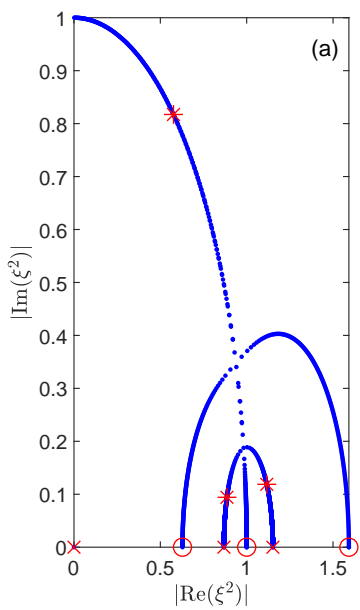
minimisation.

\subsubsection{Amplitude minimisation}

As in the case of the parallel LRLC shunt, the value of $\mu_{0}$ (and thus of $L_{0}$ ) can be set according to Eq. (41), while the other parameters must be derived solving the overdetermined system of three equations and five unknowns. Therefore, also in this case, the values of $\beta$ and $\kappa$ need to be set by a numerical
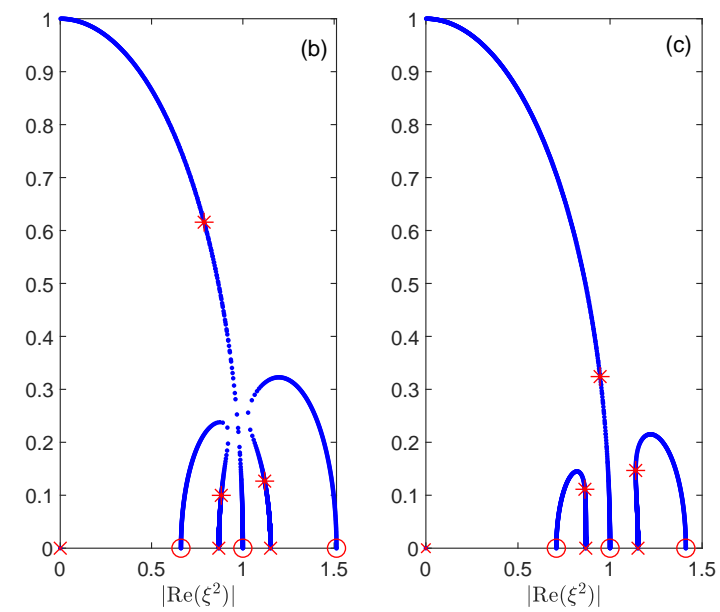

Figure 9: Trends of the absolute values of the real and imaginary parts of the squared roots (LRLC in series layout): $\kappa=5 \kappa_{0}(\mathrm{a}), \kappa=10 \kappa_{0}$ (b) and $\kappa=20 \kappa_{0}$ (c). The value of $\kappa_{0}$ is 0.02 .

The value of $\beta$ is obtained from the same criterion as before, looking for the minimisation of the peak of the dynamic amplification for a given value of $\kappa$ relative to $\kappa_{0}$. To set the $\kappa$ value, it is again possible to look at the system root locus. Figure 9 shows, for the series LRLC, the trajectories of the squared roots in the complex plane for three different values of $\kappa / \kappa_{0}$ with respect to varying values of $\beta$. The crosses represent the roots when $\beta \rightarrow \infty$, while the circles are the roots when $\beta$ is null. Moreover, the asterisks are related to the optimal $\beta$ value. Also in this case, a condition where the complex poles follow separated trajectories is achievable by increasing the $\kappa$ value above a threshold 

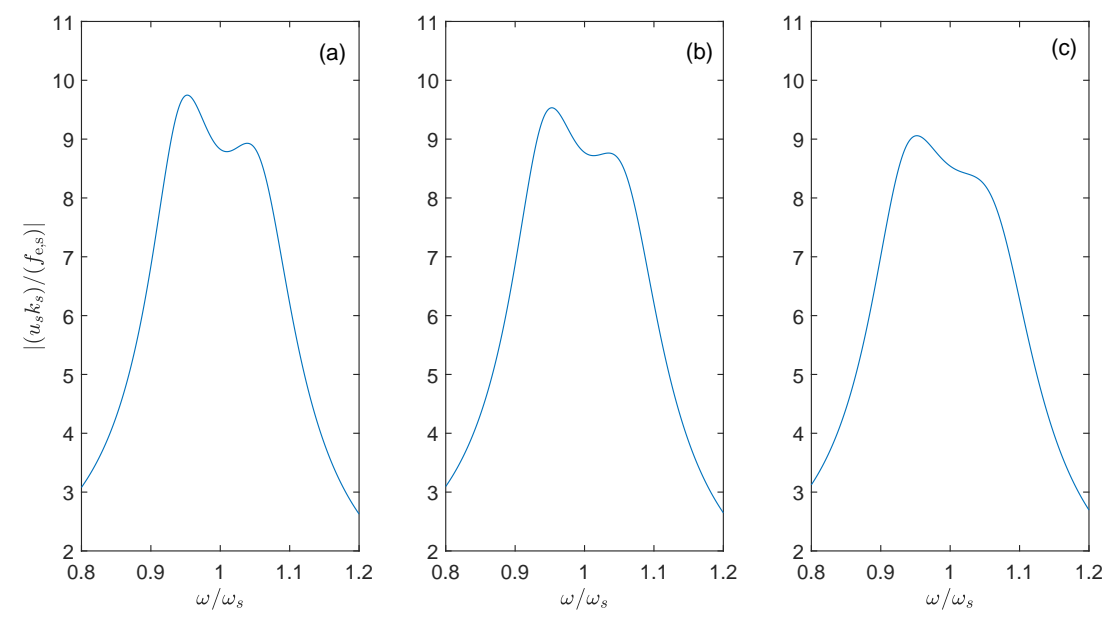

Figure 10: Amplitude of the FRF (LRLC in series layout, see Eq. (35)): $\kappa=5 \kappa_{0}(\mathrm{a}), \kappa=10 \kappa_{0}$ (b) and $\kappa=20 \kappa_{0}$ (c). The value of $\kappa_{0}$ is 0.02 and the $\beta$ value is set according to the $\mathrm{H}_{\infty}$-norm optimisation (see the asterisks in Fig. 9).

value represented by the bifurcation of the roots (see Fig. 9b). By looking at the optimal positions of the roots in Fig. 9 (i.e. the asterisks) and their corresponding FRF amplitudes in Fig. 10, it is found that the situation characterised by two separated side lobes provides the highest attenuation level and the desired separation of the roots relative to the bifurcation point. Therefore, the ratio $\kappa / \kappa_{0}=20$ secures the desired damping and response mitigation, as shown in Figs. 9c and 10c.

\subsubsection{Bifurcation point}

As in the case of the parallel LRLC, it is possible to find the threshold value of $\kappa=\kappa_{\mathrm{thr}, \mathrm{s}}$, above which the separation of the side lobes in Fig. 9c is guaranteed. This threshold value is the $\kappa$ value for which it is possible to have the bifurcation point, where all the roots exhibit the same frequency and damping and lie on the circumference with a unitary radius in the complex $\xi^{2}$-plane. This condition is achieved if the poles of the system, expressed by Eq. (37), satisfy the requirement of Eq. (33). Therefore, by equating the first, second and third order terms of these two equations and then applying the equal 
modal damping conditions in Eq. (41), the value of $\kappa_{\mathrm{thr}, \mathrm{s}}$ becomes:

$$
\kappa_{\mathrm{thr}, \mathrm{s}}=\frac{1}{8}
$$

It should be noticed that in this case the value of $\kappa$ must be increased above this value for the two desired individual loci to appear (as in Fig. 9c). Conversely, in the parallel case, a decrease of the $\kappa$ value leads to the desired separation of the loci. According to Eqs. (32) and (41), this implies that the optimal values of the inductance $L$ will be larger in the series than in the parallel LRLC shunt.

\subsection{The optimal value of $\kappa$}

Sections 3.1 and 3.2 showed that the optimal value of $\kappa$ must be sought in a range of values either below or above a certain bifurcation threshold, according to the circuit layout considered (parallel or series). The optimal $\kappa$ value will be in the considered range that guarantees the maximum attenuation level when the corresponding optimal $\beta$ value minimises the $\mathrm{H}_{\infty}$-norm of the FRF amplitude in Eqs. (20) or (35). For the case of the parallel LRLC, the value of $\kappa$ must be smaller than $\kappa_{\mathrm{thr}, \mathrm{p}}$, and thus obtained between zero and $\kappa_{\mathrm{thr}, \mathrm{p}}$. The problem is more complicated in the case of the series LRLC. Indeed, from the analysis performed in Section 3.2, it has emerged that the value of $\kappa$ must be larger than $\kappa_{\mathrm{thr}, \mathrm{s}}$ but no information is available regarding a possible upper bound. However, it is possible to find the optimal value of $\kappa$ searching in a range such that the value of $1 / \kappa$ is between zero and $1 / \kappa_{\mathrm{thr}, \mathrm{s}}$, which is numerically more convenient.

Section 4 will show a numerical simulation where the classical LR shunt with balanced calibration and the new LRLC shunt will be compared in terms of attenuation performance and robustness with respect to potential mistuning.

\section{The performances of the LRLC shunt}

This section presents a numerical analysis in which the LRLC shunt is compared to the classical balanced calibration of the LR shunt in terms of vibration 


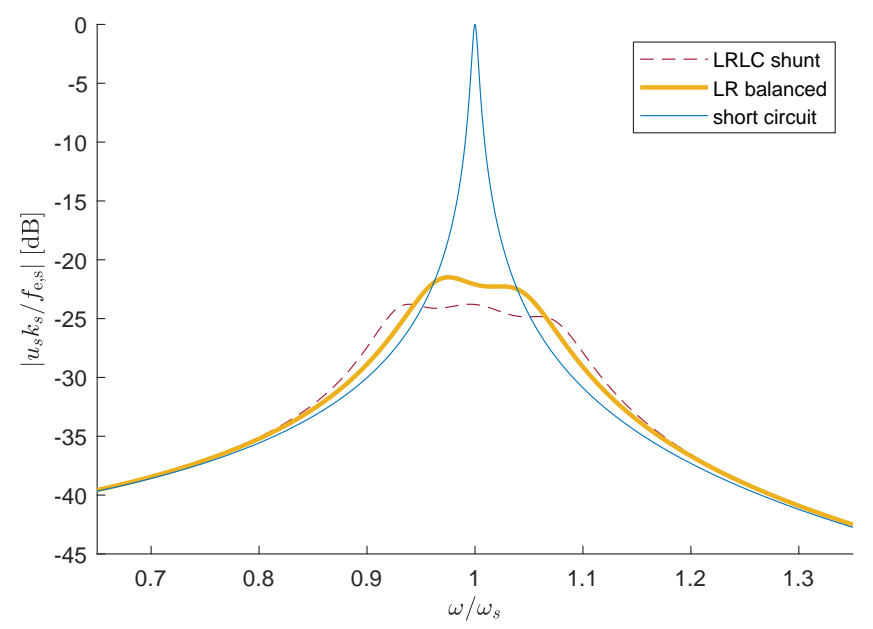

Figure 11: FRF amplitude. $\kappa=40 \kappa_{0}$ for the LRLC shunt. Series configuration for both the LRLC and LR shunts.

Table 2: Values of the system parameters

\begin{tabular}{cccccc}
\hline$\omega_{s} /(2 \pi)[\mathrm{Hz}]$ & $\hat{\omega}_{s} /(2 \pi)[\mathrm{Hz}]$ & $\zeta_{s}$ & $\sqrt{\kappa_{0}}$ & $C_{s}[\mathrm{nF}]$ & $\left|\theta_{s}\right|\left[\mathrm{kg}^{-(1 / 2)} \mathrm{NV}^{-1}\right]$ \\
\hline 100.00 & 100.50 & $3.0 \cdot 10^{-3}$ & 0.1 & 40.0 & 0.0126 \\
\hline
\end{tabular}

attenuation. This comparison has been carried out for both perfect tuning and mistuning. Indeed, the situation of mistuning is likely to be faced in real applications due to changes of the parameters of either the shunt impedance or the primary system to be damped by, for example, thermal shifts. The performance analysis in mistuned conditions also allows to validate the overall robustness of the proposed LRLC shunt.

The analysis is presented here considering a specific system chosen as an example, whose characteristics are gathered in Tab. 2. Nevertheless, the outcomes of the analysis can be generalised to any mechanical system equipped with a piezoelectric actuator; just a single example is shown here for the sake of conciseness. Figure 11 shows the amplitude of the FRF in perfect tuning for the LRLC shunt (series layout) with $\kappa=40 \kappa_{0}$. This value is very close, but not exactly equal, to the optimal value of $\kappa$. However, this difference is negligible in terms of attenuation performance, as the attenuations achieved by $\kappa=35 \kappa_{0}$ 


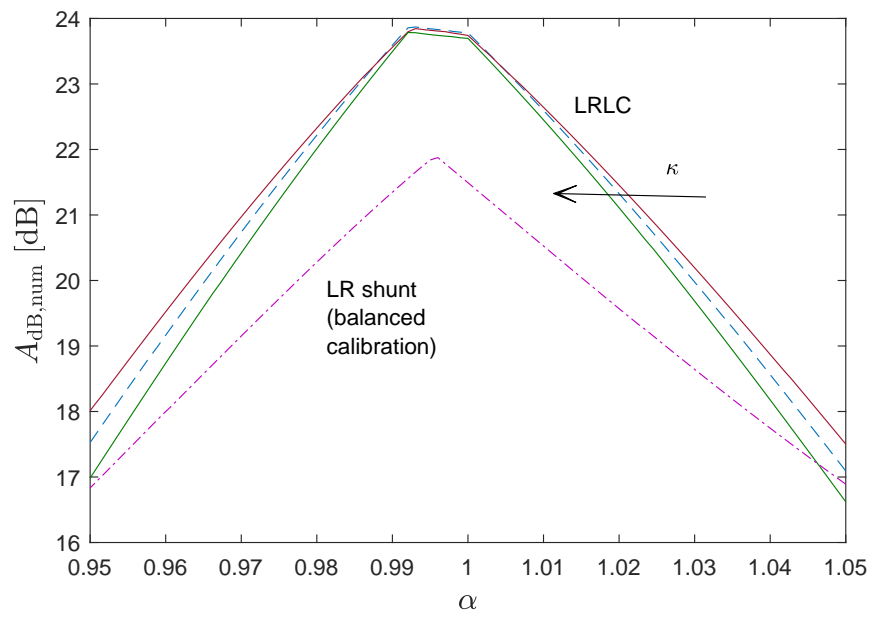

Figure 12: The value of $A_{\mathrm{dB}}$,num as a function of $\alpha$. The values used for $\kappa$ in the LRLC shunt are: $35 \kappa_{0}, 40 \kappa_{0}$ and $45 \kappa_{0}$. Series configuration for both the LRLC and LR shunts.

and $\kappa=45 \kappa_{0}$ are marginally worse than that for $\kappa=40 \kappa_{0}$. Therefore, a more detailed search for the actual optimum of $\kappa$ is practically useless. This point will be addressed again at the end of this section.

Looking at the curve related to the LRLC shunt in Fig. 11, it can be noticed that the presence of an additional resonance (compared to the classical LR shunt) characterises the proposed circuit and provides an improvement in terms of attenuation performance over the traditional LR shunt. Figure 11 shows the FRF amplitudes for both the LRLC and the LR shunt with balanced calibration and in series layout, clearly indicating the improved performance in terms of $\mathrm{H}_{\infty}$ control of the LRLC shunt, with an increase in attenuation of approximately $2.3 \mathrm{~dB}$ for this specific case.

Considering the robustness analysis with respect to possible mistuning of the shunt, a parameter $\alpha$ has been used and defined as:

$$
\alpha=\frac{\omega_{\text {cal }}}{\omega_{s}}
$$

where $\omega_{\text {cal }}$ is the frequency to which the shunt impedance has been tuned. The parameter $\alpha$ thus represents the amount of mistuning experienced by the system. 
Figure 12 shows the attenuation value as a function of $\alpha$, where the attenuation is expressed as:

$$
A_{\mathrm{dB}, \text { num }}=20 \log _{10} \frac{H_{\mathrm{sc}}}{H_{\text {shunt,num }}}
$$

where $H_{\mathrm{sc}}$ is the maximum of the amplitude of the system FRF with the piezoelectric actuator short-circuited, while $H_{\text {shunt,num }}$ is the maximum of the amplitude of the system FRF with the considered shunt circuit in the simulated mistuned condition.

Figure 12 shows that the LRLC shunt improves the attenuation compared to the LR shunt, even in presence of mistuning for reasonable values of $\alpha$. The higher robustness of the LRLC shunt is demonstrated by the trend of the curves for $\alpha$ values between 0.99 and 1. Indeed, in this range, the LRLC shunt curves show an almost flat plateau, while the classical LR shunt exhibits steep curves with a very local optimum. Therefore, the LRLC shunt appears more robust than the LR shunt, thanks to the additional resonance introduced by the new circuit, which allows to obtain a wider and flatter shape of the FRF of the controlled system. It is also noticed that a range of $\alpha$ of approximately $1 \%$, which corresponds to the plateau of the LRLC curves, is close to the natural uncertainty that can be encountered in real applications and to the possible bias effects due to, for example, environmental changes. Furthermore, the width of the flat plateau increases significantly when the value of $\kappa_{0}$ increases, as evidenced with the system used in the experiments of Section 6 . Therefore, when the characteristics of the piezoelectric actuator are optimised for controlling a given mode, which is a reasonable assumption in practical applications, the coupling factor is large [46] and the LRLC shunt becomes consistently more robust than the corresponding LR shunt.

Furthermore, it is noted that, if it is desired to lift the LRLC curves of Fig. 12 far from $\alpha=1$, it is sufficient to slightly decrease the value of $\kappa$ (using the corresponding optimal value of $\beta$ ), accepting a slight (and often negligible) decrease in attenuation for $\alpha=1$. In addition, this increases the robustness of 
the shunt due to a corresponding reduced slope of the curves.

Figure 12 also shows that for a value of $\kappa=35 \kappa_{0}$ (thus, lower than the value of $\kappa=40 \kappa_{0}$ used in Fig. 11) and for a value of $\kappa=45 \kappa_{0}$ (thus a higher value than in Fig. 11), the attenuation performance for $\alpha=1$ (i.e. perfect tuning) is sufficiently close to that achieved with $\kappa=40 \kappa_{0}$, which is therefore considered optimal, as mentioned at the beginning of this section.

Outcomes similar to those presented so far for the series layouts are also found in case of a comparison between the classical parallel LR shunt and the new LRLC shunt in its parallel layout.

The tuning methods for both the LR and LRLC shunts are developed under the hypothesis of low modal coupling, as mentioned in Section 2. This means that the contribution of the out-of-band modes to the mechanical behaviour of the electro-mechanical system dynamics is neglected. Instead, the contribution of the out-of-band modes is taken into account in the electrical behaviour of the electro-mechanical system by the term with $C_{s}$ in the model of Section 2 . However, it is important to underline that in case the contribution of the out-ofband modes to the mechanical part of the electro-mechanical system dynamics is not negligible, because they are close in frequency to the target mode, the effects on the attenuation provided by the two different shunts is expected to be similar. Therefore, this additional effect will not affect the results of the comparison between the two shunt impedances and the outcome of the analysis. Moreover, looking at Fig. 11, it is evident that the FRFs related to the two different shunts differ in a frequency range of about \pm 10 to $20 \%$ of $\omega_{s}$ (see also Section 6). Therefore, the out-of-band modes can change the results of the comparison between the LR and LRLC shunts only in case they are very close to the targeted mode, whereby the low modal coupling hypothesis (which is the foundation of the proposed method) is not applicable anymore. However, since the robustness of the LRLC shunt is higher than that of the LR shunt, the LRLC shunt is expected to still provide a higher attenuation level compared to the LR shunt, even in case of high influence from the out-of-band modes. Finally, it is worth evidencing that even in the case the hypothesis of low modal 


\section{Attenuation by the LRLC shunt}

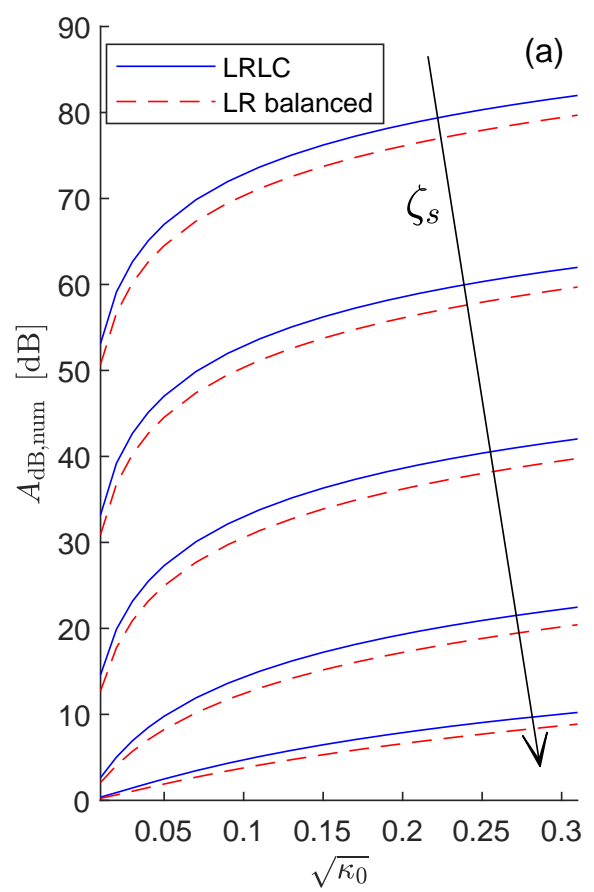

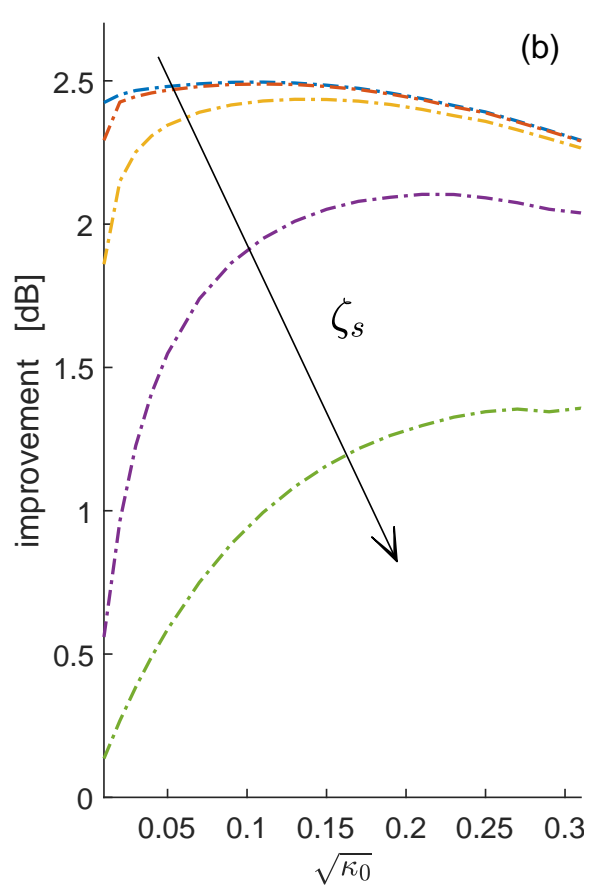

Figure 13: The value of $A_{\mathrm{dB}}$,num as a function of $\sqrt{\kappa_{0}}$ for different values of $\zeta_{s}\left(10^{-5}, 10^{-4}\right.$, $10^{-3}, 10^{-2}$ and $5 \cdot 10^{-2}$ ) (a) and the corresponding attenuation improvement provided by the

LRLC shunt (b). Series configuration for both the LRLC and LR shunts.

coupling is not satisfied, the result of the proposed LRLC shunt optimisation procedure (as well as in the case of an LR shunt) can be used as the starting point for a minimisation aimed at tuning the LRLC impedance using a multidegree-of-freedom model like that described in [15].

The results shown in Fig. 11, obtained for a system chosen as an example, can be generalised noticing that the FRFs associated to the classical LR shunt (in normalised form using $r$ in place of $\omega$ in Eq. (8)) and the new LRLC shunt (see Eq. (35)) are dependent on only two parameters of the electromechanical system: $\kappa_{0}$ and $\zeta_{s}$. Therefore, it is possible to numerically compute the attenuation provided by the two different impedance layouts as a function 
Table 3: Values of the experimental parameters

\begin{tabular}{cccccc}
\hline$\omega_{s} /(2 \pi)[\mathrm{Hz}]$ & $\hat{\omega}_{s} /(2 \pi)[\mathrm{Hz}]$ & $\zeta_{s}$ & $\sqrt{\kappa_{0}}$ & $C_{s}[\mathrm{nF}]$ & $\left|\theta_{s}\right|\left[\mathrm{kg}^{-(1 / 2)} \mathrm{NV}^{-1}\right]$ \\
\hline 34.29 & 35.44 & $4.5 \cdot 10^{-3}$ & 0.2602 & 39.92 & 0.0112 \\
\hline
\end{tabular}

of $\kappa_{0}$ and $\zeta_{s}$. For $\kappa_{0}$, the authors chose to consider values from $0.01^{2}$ (very low value) to $0.31^{2}$ (close to the largest value encountered in practice [46]). For $\zeta_{s}$, the authors have used five different values (from very low to very high for typical mechanical systems).

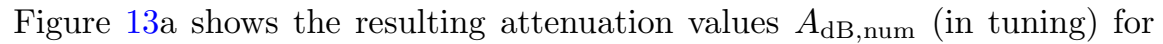
the two different shunts. Usually, the LRLC shunt allows to have attenuation improvements between 2 and $2.5 \mathrm{~dB}$ (see Fig. 13b), except for low values of $\kappa_{0}$ coupled to high values of $\zeta_{s}$ where the improvement decreases. However, this improvement is still significant since also the overall attenuation decreases in this case. Therefore, Fig. 13 can be used as an abacus for predicting the improvement provided by the LRLC shunt compared to the classical LR shunt with equal modal damping calibration.

The improvement provided by the LRLC shunt is most of the time larger than $2 \mathrm{~dB}$, and thus, in percentage, the LRLC shunt allows to further decrease the peak of the FRF amplitude of more than 25\% (and many times of more than 30\%). This result is in accordance with the results usually obtained from multiple-TVA configurations when compared to situations where a single TVA is used (e.g. [40, 43]). The result is remarkable, especially in light of the fact that the improvement is obtained by a completely passive approach. However, it is important to remember that, in case operational amplifiers (OP-AMP) are needed to build the inductances of the shunt circuit because of their high values (see Section 6), the approach can be considered as passive from a dynamical point of view, although not strictly passive with respect to power consumption (i.e. OP-AMPs need a power supply). 


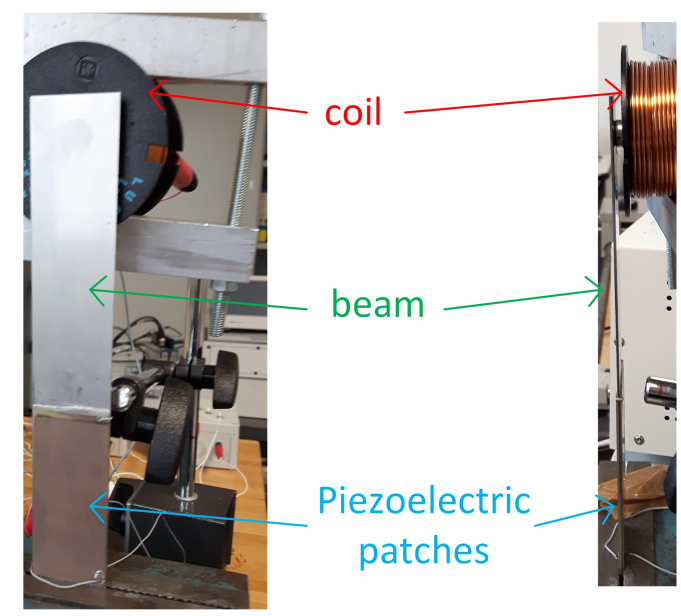

Figure 14: The experimental set-up.

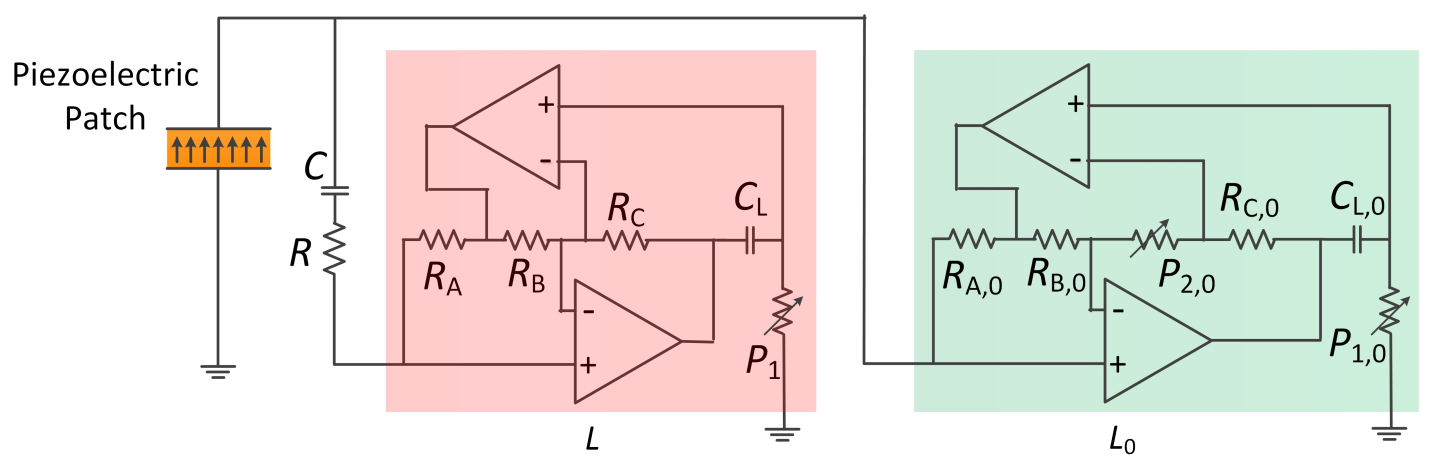

Figure 15: The electrical layout used for the LRLC shunt.

\section{Experiments}

This section presents the experimental tests carried out with the aim of validating the theoretical outcomes shown previously. The set-up used was made from a stainless steel cantilever beam with a length equal to $180 \mathrm{~mm}$, a width of $30.5 \mathrm{~mm}$ and a thickness of $1.1 \mathrm{~mm}$. Two piezoelectric patches (length $70 \mathrm{~mm}$, width $30.0 \mathrm{~mm}$ and thickness $0.55 \mathrm{~mm}$, material PIC 151) were bonded at the clamped end of the beam (one on each side of the beam) and electrically connected in series. The system was forced by means of a contactless magnetic 


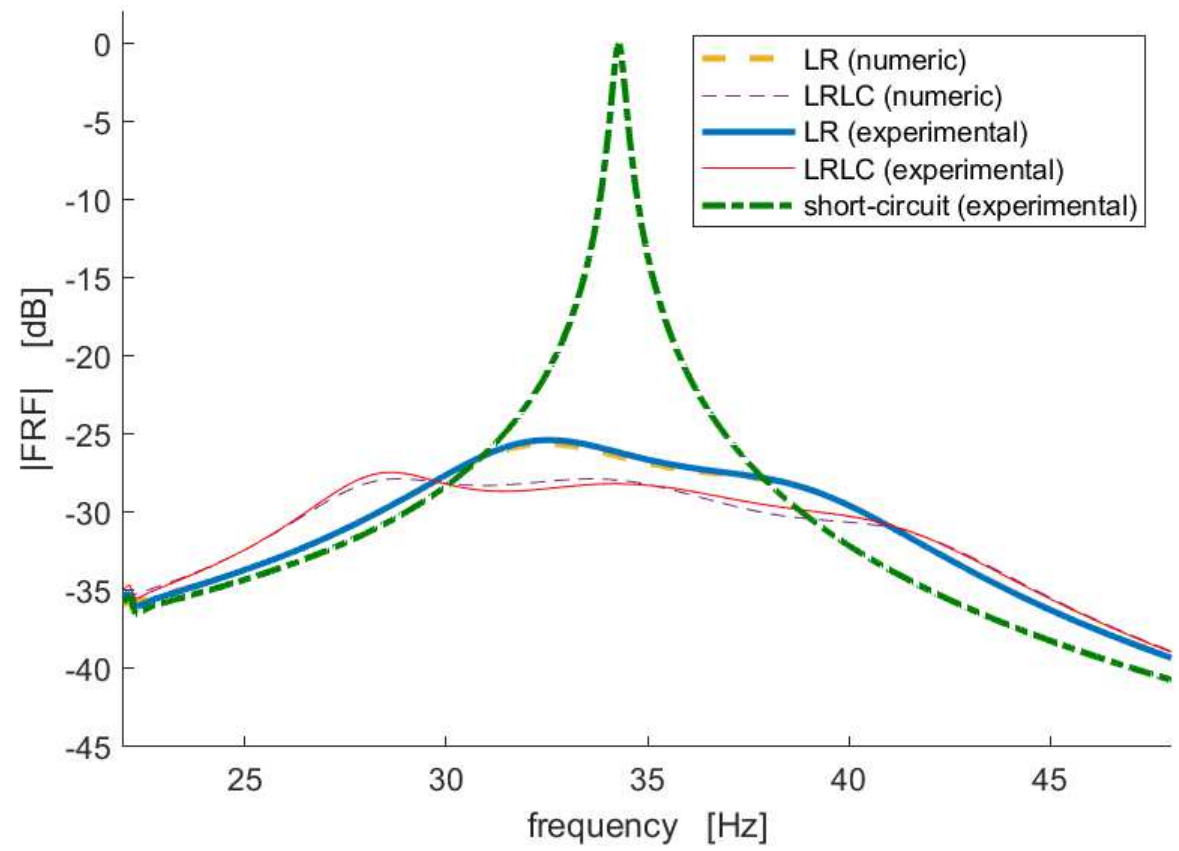

Figure 16: Experimental and numerical FRF amplitudes (in terms of displacement over force) in short-circuit and in tuned condition.

actuator [51] and the corresponding vibration response was collected by using a laser velocimeter. This set-up, shown in Fig. 14, is the same already used in [18], from which interested readers can find more details.

The first mode of the structure was considered for the tests because its amplitude was higher than that of the other modes in the low frequency range. Its eigenfrequencies with the patches in short- $\left(\omega_{\mathrm{s}}\right)$ and open-circuit $\left(\hat{\omega}_{\mathrm{s}}\right)$, as well as the mechanical non-dimensional damping ratio $\zeta_{s}$, were identified by means of

Table 4: Values of the electrical parameters of the shunt impedances

\begin{tabular}{ccccc}
\hline type of shunt & $L[\mathrm{H}]$ & $R[\mathrm{k} \Omega]$ & $C[\mathrm{nF}]$ & $L_{0}[\mathrm{H}]$ \\
\hline LR shunt (balanced calibration) & 472.9 & 38.89 & - & - \\
\hline LRLC & 3100.7 & 367.0 & 6.95 & 539.7 \\
\hline
\end{tabular}




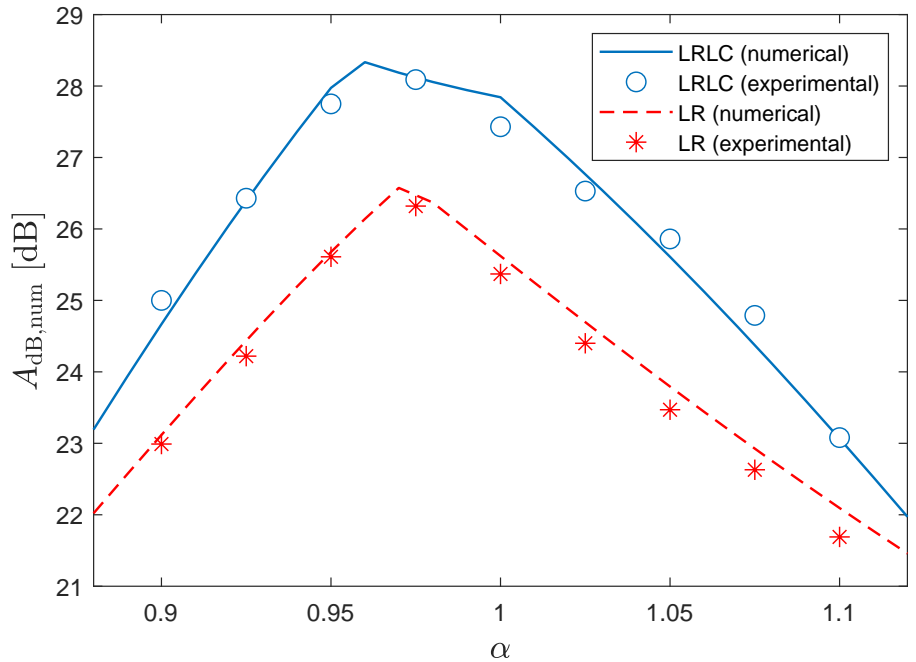

Figure 17: Experimental and numerical attenuation as a function of $\alpha$.

an experimental modal analysis (see Tab. 3). The value of $\theta_{s}$ was estimated by means of Eq. (13), where knowledge of the $C_{s}$ value is required. This value was estimated by measuring the capacitance of the piezoelectric patch as a function of $\omega$ with an LCR meter (see [18] for more details). All the estimated system parameters are gathered in Tab. 3.

The classical LR shunt with balanced calibration (see Section 2.1) and the new LRLC shunt (see Section 3) were compared in series layout. All the inductances were built using OP-AMPs with the Antoniou's circuit [6, 52] because of the high inductance values required. The whole electrical layout employed for the LRLC impedance is shown in Fig. 15. It is noticed that the layout of $L_{0}$ is different from that of $L$. Indeed, in the circuit of $L_{0}$, there is the variable resistance $P_{2,0}$, which is used to compensate for the parasitic resistances usually present when employing OP-AMPs to build inductances [6]. The presence of this potentiometer for $L_{0}$ is important because $L_{0}$ is the only element on its branch (see Fig. 2d) and thus the parasitic resistance must be minimised. The value of $P_{2,0}$ was set in order to have a parasitic resistance slightly positive and not exactly null, as this would have increased the risk of instabilities from e.g. 
a thermal shift imposing a negative parasitic resistance. The value of the parasitic resistance was estimated close to $500 \Omega$. Numerical simulations showed that such a resistance would not cause significant changes in the attenuation performance compared to the ideal case without parasitic resistance. The variable resistance $P_{2,0}$ was not necessary for $L$ because this inductance is in series with the resistance $R$ and thus it was easy to compensate the presence of the parasitic resistance by changing the value of $R$ accordingly. All the OP-AMPs (OPA 445 type) were supplied with a constant voltage of $\pm 30 \mathrm{~V}$.

Figure 16 shows the numerical and experimental FRF amplitudes (in terms of displacement over force) in the tuned condition for the classical LR shunt with balanced calibration and the new LRLC impedance. The match between experimental and numerical data is good. Furthermore, the LRLC shunt achieves, as expected, a higher attenuation performance over the classical LR shunt (experimental improvement of approximately $2.1 \mathrm{~dB}$ ). The values of the various electrical parameters used for the shunts are provided in Tab. 4. It is noticed that the value of the capacitance $C$ for the LRLC shunt is not optimal (optimal value is $7.36 \mathrm{nF}$ and used value is $6.95 \mathrm{nF}$ ). The use of this non-optimal value was due to the available capacitors in the laboratory. However, this difference does not cause any significant change in terms of vibration attenuation (as evidenced by numerical simulations) and thus it was considered acceptable.

In order to validate the results related to the robustness of the LRLC shunt, also tests in mistuned conditions were performed, as shown in Fig. 17. This figure presents the value of the attenuation $A_{\mathrm{dB}, \text { num }}$ as a function of the mistuning index $\alpha$ (see Eq. (43)). It is worth highlighting that there is a difference in the way of causing mistuning in the experiments with respect to what has been described in Section 4, where the mistuning was obtained by simulating a shift of the actual eigenfrequency value of the system (i.e. simulating a plausible real situation). Conversely, the mistuning is here obtained by changing the frequency value to which the shunt is optimised, while the actual eigenfrequency of the structure does not change. However, the meaning of the coefficient $\alpha$ does not differ and it is still an index of mistuning. As can be noticed by looking at the 
experimental points in Fig. 17, also in this case there is a good match between numerical and experimental results. Moreover, the improved performance of the new LRLC shunt is experimentally demonstrated, as the LRLC shunt provides higher attenuation values compared to the classical LR shunt for all the tested values of $\alpha$. The higher robustness of the LRLC shunt is also demonstrated by the trend of the theoretical curves in Fig. 17 for $\alpha$ values between 0.95 and 1, within which the LRLC shunt curve has an almost flat plateau, while the classical LR shunt exhibits steep curves with a very local optimum.

\section{Conclusion}

The paper has presented a new type of impedance to be employed when mono-modal vibration control is carried out with a piezoelectric shunt. The new impedance can have two different layouts. However, in both cases the driving idea is that the shunt impedance must be such that it generates two different resonances. To this purpose, it is composed of two inductances, one capacitance and one resistance, comprising the resulting LRLC shunt.

Guidelines are provided for setting all the electrical parameters of the shunt impedance. The new shunt network is found to be reliable and to provide better attenuation performance than the classical LR shunt based on balanced calibration. The benefits of the newly proposed impedance are evident in both tuned and mistuned situations.

The theoretical outcomes have been validated by means of a test set-up in which the inductances used for the shunt have been built by OP-AMPs. Good agreement between theory and experiments has been obtained, validating the proposed shunt concept. A future study should address the power consumption by the augmented LRLC shunt when using OP-AMPs to build inductances.

\section{References}

[1] C. Bricault, C. Pézerat, M. Collet, A. Pyskir, P. Perrard, G. Matten, V. Romero-Garcia, Multimodal reduction of acoustic radiation of thin 
plates by using a single piezoelectric patch with a negative capacitance shunt, Applied Acoustics 145 (2019) 320-327.

[2] P. Gardonio, D. Casagrande, Shunted piezoelectric patch vibration absorber on two-dimensional thin structure: tuning considerations, Journal of Sound and Vibration 395 (2017) 26-47.

[3] R. Darleux, B. Lossouarn, J.-F. Deü, Passive self-tuning inductor for piezoelectric shunt damping considering temperature variations, Journal of Sound and Vibration 432 (2018) 105-118.

[4] J. F. Toftekaer, A. Benjeddou, J. Høgsberg, S. Krenk, Optimal piezoelectric resistive-inductive shunt damping of plates with residual mode correction, Journal of Intelligent Material Systems and Structures 29 (16) (2018) 33463370.

[5] N. Hagood, A. von Flotow, Damping of structural vibrations with piezoelectric materials and passive electrical networks, Journal of Sound and Vibration 146 (1991) 243-268.

[6] O. Thomas, J. Ducarne, J. Deü, Performance of piezoelectric shunts for vibration reduction, Smart Materials and Structures 21 (1) (2012) Article ID 015008 .

[7] P. Soltani, G. Kerschen, G. Tondreau, A. Deraemaeker, Tuning of a piezoelectric vibration absorber attached to a damped structure, Journal of Intelligent Material Systems and Structures 28 (9) (2017) 1115-1129.

[8] K. Yamada, H. Matsuhisa, H. Utsuno, K. Sawada, Optimum tuning of series and parallel lr circuits for passive vibration suppression using piezoelectric elements, Journal of Sound and Vibration 329 (24) (2010) 5036-5057.

[9] V. Matveenko, N. Iurlova, D. Oshmarin, N. Sevodina, M. Iurlov, An approach to determination of shunt circuits parameters for damping vibrations, International Journal of Smart and Nano Materials 9 (2) (2018) 135149. 
[10] G. Caruso, A critical analysis of electric shunt circuits employed in piezoelectric passive vibration damping, Smart Materials and Structures 10 (5) (2001) 1059-1068.

[11] M. Berardengo, O. Thomas, C. Giraud-Audine, S. Manzoni, Improved shunt damping with two negative capacitances: an efficient alternative to resonant shunt, Journal of Intelligent Material Systems and Structures 28 (16) (2017) 2222-2238.

[12] M. Berardengo, S. Manzoni, O. Thomas, C. Giraud-Audine, A new electrical circuit with negative capacitances to enhance resistive shunt damping, in: Proceedings of the ASME 2015 Conference on Smart Materials, Adaptive Structures and Intelligent Systems - SMASIS 2015 - September 21-23, 2015 - Colorado Springs (CO, USA), 2015.

[13] S. Behrens, A. J. Fleming, S. O. R. Moheimani, A broadband controller for shunt piezoelectric damping of structural vibration, Smart Materials and Structures 12 (1) (2003) 18-28.

[14] B. S. Beck, K. A. Cunefare, M. Collet, Response-based tuning of a negative capacitance shunt for vibration control, Journal of Intelligent Material Systems and Structures 25 (13) (2014) 1585-1595.

[15] M. Berardengo, S. Manzoni, A. Conti, Multi-mode passive piezoelectric shunt damping by means of matrix inequalities, Journal of Sound and Vibration 405 (2017) 287-305.

[16] A. Fleming, S. Behrens, S. Moheimani, Reducing the inductance requirements of piezoelectric shunt damping systems, Smart Materials and Structures 12 (1) (2003) 57-64.

[17] M. Neubauer, R. Oleskiewicz, K. Popp, T. Krzyzynski, Optimization of damping and absorbing performance of shunted piezo elements utilizing negative capacitance, Journal of Sound and Vibration 298 (1-2) (2006) 84107. 
[18] M. Berardengo, S. Manzoni, O. Thomas, M. Vanali, Piezoelectric resonant shunt enhancement by negative capacitances: optimisation, performance and resonance cancellation, Journal of Intelligent Material Systems and Structures 29 (12) (2018) 2581-2606.

[19] M. Berardengo, A. Cigada, S. Manzoni, M. Vanali, Vibration control by means of piezoelectric actuators shunted with lr impedances: Performance and robustness analysis, Shock and Vibration 2015 (2015) Article ID 704265 .

[20] A. Preumont, Mechatronics: Dynamics of Electromechanical and Piezoelectric Systems, Springer, 2006.

[21] J. Høgsberg, S. Krenk, Balanced calibration of resonant shunt circuits for piezoelectric vibration control, Journal of Intelligent Material Systems and Structures 23 (17) (2012) 1937-1948.

[22] J. Høgsberg, S. Krenk, Balanced calibration of resonant piezoelectric rl shunts with quasi-static background flexibility correction, Journal of Sound and Vibration 341 (2015) 16-30.

[23] M. Berardengo, S. Manzoni, M. Vanali, The behaviour of mistuned piezoelectric shunt systems and its estimation, Shock and Vibration 2016 (2016) Article ID 9739217.

[24] U. Andreaus, M. Porfiri, Effect of electrical uncertainties on resonant piezoelectric shunting, Journal of Intelligent Material Systems and Structures 18 (2007) 477-485.

[25] L. Zuo, S. Nayfeh, The two-degree-of-freedom tuned-mass damper for suppression of single-mode vibration under random and harmonic excitation, Journal of Vibration and Acoustics 128 (2006) 56-65.

[26] L. Zuo, S. Nayfeh, Minimax optimization of multi-degree-of-freedom tunedmass dampers, Journal of Sound and Vibration 272 (2004) 893-908. 
[27] S. Elias, V. Matsagar, Research developments in vibration control of structures using passive tuned mass dampers, Annual Reviews in Control 44 (2017) 129-156.

[28] S. Elias, V. Matsagar, T. Datta, Distributed tuned mass dampers for multimode control of benchmark building under seismic excitations, Journal of Earthquake Engineering 23 (7) (2019) 1137-1172.

[29] R. Debbarma, S. Hazari, Mass distribution of multiple tuned mass dampers for vibration control of structures under earthquake load, International Journal of Emerging Technology and Advanced Engineering 3 (8) (2013) 198-202.

[30] Y. Luo, H. Sun, X. Wang, L. Zuo, N. Chen, Wind induced vibration control and energy harvesting of electromagnetic resonant shunt tuned massdamper-inerter for building structures, Shock and Vibration 2017 (2017) article ID: 4180134.

[31] E. Mrabet, M. Ichchou, N. Bouhaddi, Random vibro-acoustic control of internal noise through optimized tuned mass dampers, Mechanical Systems and Signal Processing 130 (2019) 17-40.

[32] L. Zuo, Effective and robust vibration control using series multiple tunedmass dampers, Journal of Vibration and Acoustics 131 (2009) article ID: 031003.

[33] T. Asami, Optimal design of double-mass dynamic vibration absorbers arranged in series or in parallel, Journal of Vibration and Acoustics 139 (2017) article ID. 011015.

[34] T. Asami, Y. Mizukawa, T. Ise, Optimal design of double-mass dynamic vibration absorbers minimizing the mobility transfer function, Journal of Vibration and Acoustics 140 (2018) article ID. 061012. 
[35] L. Zuo, S. Nayfeh, Optimization of the individual stiffness and damping parameters in multiple-tuned-mass-damper systems, Journal of Vibration and Acoustics 127 (2005) 77-83.

[36] C. Li, Optimum multiple tuned mass dampers for structures under the ground acceleration based on ddmf and admf, Earthquake Engineering and Structural Dynamics 31 (2002) 897-919.

[37] K. Xu, T. Igusa, Dynamic characteristics of multiple substructures with closely spaced frequencies, Earthquake Engineering and Structural Dynamics 21 (1992) 1059-1070.

[38] S. Bakre, R. Jangid, Optimum multiple tuned mass dampers for baseexcited damped main system, International Journal of Structural Stability and Dynamics 4 (4) (2004) 527-542.

[39] A. Javidialesaadi, N. Wierschem, Optimal design of rotational inertial double tuned mass dampers under random excitation, Engineering Structures 165 (2018) 412-421.

[40] E. Barredo, J. Mendoza-Larios, J. Mayén, A. Flores-Hernández, J. Colín, M. Arais-Montiel, Optimal design for high-performance passive dynamic vibration absorbers under random vibration, Engineering Structures 195 (2019) 469-489.

[41] J. Yang, S. Sun, J. Chi, D. Ning, H. Du, S. Zhang, W. Li, S. Mao, Development and evaluation of an mrebased absorber with two individually controllable natural frequencies, Smart Materials and Structures 27 (2018) article ID: 095002.

[42] Y. Liu, C.-C. Lin, J. Parker, L. Zuo, Exact h2 optimal tuning and experimental verification of energy-harvesting series electromagnetic tuned-mass dampers, Journal of Vibration and Acoustics 138 (2016) article ID. 061003. 
[43] L. Zuo, W. Cui, Dual-functional energy-harvesting and vibration control: Electromagnetic resonant shunt series tuned mass dampers, Journal of Vibration and Acoustics 135 (5) (2013) article ID: 051018.

[44] M. Berardengo, O. Thomas, C. Giraud-Audine, S. Manzoni, Improved resistive shunt by means of negative capacitance: new circuit, performances and multi mode control, Smart Materials and Structures 25 (2016) Article ID 075033 .

[45] J. Høgsberg, S. Krenk, Calibration of piezoelectric rl shunts with explicit residual mode correction, Journal of Sound and Vibration 386 (2017) 65-81.

[46] J. Ducarne, O. Thomas, J. Deü, Placement and dimension optimization of shunted piezoelectric patches for vibration reduction, Journal of Sound and Vibration 331 (14) (2012) 3286-3303.

[47] O. Thomas, J.-F. Dë̈, J. Ducarne, Vibration of an elastic structure with shunted piezoelectric patches: efficient finite-element formulation and electromechanical coupling coefficients, International Journal of Numerical Methods in Engineering 80 (2) (2009) 235-268.

[48] S. Krenk, Frequency analysis of the tuned mass damper, Journal of Applied Mechanics 72 (6) (2005) 936-942.

[49] S. Krenk, J. Høgsberg, Equal modal damping design for a family of resonant vibration control formats, Journal of Applied Mechanics 19 (9) (2013) 12941315.

[50] J. Høgsberg, Vibration control by piezoelectric proof-mass absorber with resistive-inductive shunt, Mechanics of Advanced Materials and Structures (2019) 10.1080/15376494.2018.1551587.

[51] O. Thomas, C. Touzé, A. Chaigne, Asymmetric non-linear forced vibrations of free-edge circular plates. part ii: experiments, Journal of Sound and Vibration 265 (5) (2003) 1075-1101. 
[52] L. von Wangeheim, Modification of the classical gic structure and its application to rc-oscillators, Electronics Letters 32 (1) (1996) 6-8.

\section{List of table captions}

Table 1: Conditions on the coefficients of Eq. (27).

Table 2: Values of the system parameters.

Table 3: Values of the experimental parameters.

Table 4: Values of the electrical parameters of the shunt impedances.

\section{List of figure captions}

Figure 1: Vibration attenuation by means of a piezoelectric shunt.

Figure 2: Traditional resonant shunt impedance in parallel (a) and series (b), and the new LRLC shunt impedance in parallel (c) and series (d).

Figure 3: Circuit diagram of the parallel LR shunt (a) and its mechanical equivalent (b).

Figure 4: Circuit diagram of the series LR shunt (a) and its mechanical equivalent (b).

Figure 5: Circuit diagram of the parallel LRLC shunt (a) and its mechanical equivalent (b).

Figure 6: Trends of the absolute values of the real and imaginary parts of the squared roots (LRLC in parallel layout): $\kappa=0.10 \kappa_{0}$ (a), $\kappa=0.15 \kappa_{0}$ (b) and $\kappa=0.20 \kappa_{0}(\mathrm{c})$. The value of $\kappa_{0}$ is 0.02 .

Figure 7: Amplitude of the FRF (LRLC in parallel layout, see Eq. (20)): $\kappa=0.10 \kappa_{0}(\mathrm{a}), \kappa=0.15 \kappa_{0}$ (b) and $\kappa=0.20 \kappa_{0}$ (c). The value of $\kappa_{0}$ is 0.02 and the $\beta$ value is set according to the $\mathrm{H}_{\infty}$-norm optimisation (see the asterisks in Fig. 6).

Figure 8: Circuit diagram of the series LRLC shunt (a) and its mechanical equivalent (b). 
Figure 9: Trends of the absolute values of the real and imaginary parts of the squared roots (LRLC in series layout): $\kappa=5 \kappa_{0}$ (a), $\kappa=10 \kappa_{0}$ (b) and $\kappa=20 \kappa_{0}$ (c). The value of $\kappa_{0}$ is 0.02 .

Figure 10: Amplitude of the FRF (LRLC in series layout, see Eq. (35)): $\kappa=5 \kappa_{0}(\mathrm{a}), \kappa=10 \kappa_{0}$ (b) and $\kappa=20 \kappa_{0}(\mathrm{c})$. The value of $\kappa_{0}$ is 0.02 and the $\beta$ value is set according to the $\mathrm{H}_{\infty}$-norm optimisation (see the asterisks in Fig. $9)$.

Figure 11: FRF amplitude. $\kappa=40 \kappa_{0}$ for the LRLC shunt. Series configuration for both the LRLC and LR shunts.

Figure 12: The value of $A_{\mathrm{dB}, \text { num }}$ as a function of $\alpha$. The values used for $\kappa$ in the LRLC shunt are: $35 \kappa_{0}, 40 \kappa_{0}$ and $45 \kappa_{0}$. Series configuration for both the LRLC and LR shunts.

Figure 13: The value of $A_{\mathrm{dB} \text {,num }}$ as a function of $\sqrt{\kappa_{0}}$ for different values of $\zeta_{s}\left(10^{-5}, 10^{-4}, 10^{-3}, 10^{-2}\right.$ and $\left.5 \cdot 10^{-2}\right)$. Series configuration for both the LRLC and LR shunts.

Figure 14: The experimental set-up.

Figure 15: The electrical layout used for the LRLC shunt.

Figure 16: Experimental and numerical FRF amplitudes (in terms of displacement over force) in short-circuit and in tuned condition.

Figure 17: Experimental and numerical attenuation as a function of $\alpha$. 